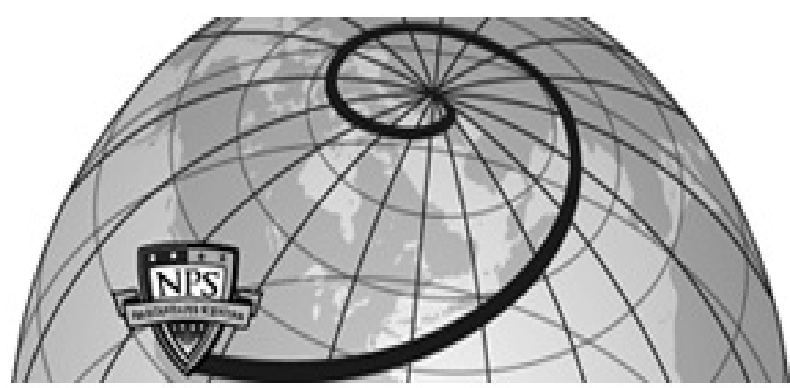

Calhoun: The NPS Institutional Archive DSpace Repository

Double-Diffusive Recipes. Part II: Layer-Merging Events

Radko, T.; Flanagan, J.D.; Stellmach, S.; Timmermans, M.-L.

Journal of Physical Oceanography, Volume 44, pp. 1285-1305, May 2014.

http://hdl.handle.net/10945/42133

This publication is a work of the U.S. Government as defined in Title 17, United States Code, Section 101. Copyright protection is not available for this work in the United States.

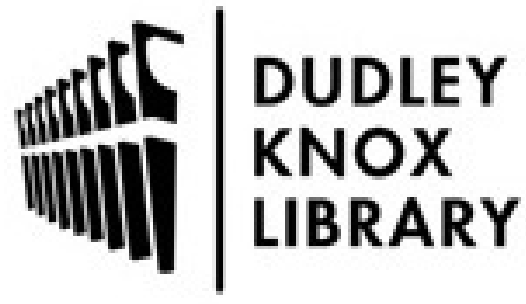

http://www.nps.edu/library
Calhoun is the Naval Postgraduate School's public access digital repository for research materials and institutional publications created by the NPS community. Calhoun is named for Professor of Mathematics Guy K. Calhoun, NPS's first appointed -- and published -- scholarly author.

Dudley Knox Library / Naval Postgraduate School 411 Dyer Road / 1 University Circle Monterey, California USA 93943 


\title{
Double-Diffusive Recipes. Part II: Layer-Merging Events
}

\author{
T. RADKO AND J. D. FLANAGAN \\ Department of Oceanography, Naval Postgraduate School, Monterey, California \\ S. STELLMACH \\ Institut für Geophysik, Westfälische Wilhelms-Universität Münster, Münster, Germany \\ M.-L. TIMMERMANS \\ Department of Geology and Geophysics, Yale University, New Haven, Connecticut
}

(Manuscript received 8 July 2013, in final form 12 December 2013)

\begin{abstract}
This study explores the dynamics of thermohaline staircases: well-defined stepped structures in temperature and salinity profiles, commonly observed in regions of active double diffusion. The evolution of staircases in time is frequently characterized by spontaneous layer-merging events. These phenomena, the authors argue, are essential in regulating the equilibrium layer thickness in fully developed staircases. The pattern and mechanics of merging events are explained using a combination of analytical considerations, direct numerical simulations, and data analysis. The theoretical merger model is based on the stability analysis for a series of identical steps and pertains to both forms of double diffusion: diffusive convection and salt fingering. The conceptual significance of the proposed model lies in its ability to describe merging events without assuming from the outset specific power laws for the vertical transport of heat and salt - the approach adopted by earlier merging models. The analysis of direct numerical simulations indicates that merging models based on the four-thirds flux laws offer adequate qualitative description of the evolutionary patterns but are less accurate than models that do not rely on such laws. Specific examples considered in this paper include the evolution of layers in the diffusive staircase in the Beaufort Gyre of the Arctic Ocean.
\end{abstract}

\section{Introduction}

One of the most dramatic signatures of active double diffusion in the ocean is related to the formation of thermohaline staircases-a series of mixed layers separated by thin stratified interfaces. While the appearance of well-defined staircases has been firmly linked to double diffusion (e.g., Schmitt 1994), the mechanics of their generation and equilibration are still debated [see the review in Radko (2013)]. Theories proposed to explain the origin of staircases include the collective instability mechanism (Stern 1969), which attributes layering to the interaction between waves and double diffusion, and the suggestion that steps in temperature and salinity profiles represent intrusions evolving into

Corresponding author address: T. Radko, Department of Oceanography, Naval Postgraduate School, 833 Dyer Road, Bldg. 232, Room 328, Monterey, CA 93943.

E-mail: tradko@nps.edu a staircase (Merryfield 2000). More recently, it has been proposed (Radko 2003, 2005) that layers form as a result of the instability of the flux-gradient laws, which was referred to as the gamma instability mechanism. This instability manifests itself in the form of growing, horizontally uniform perturbations, which transform the smooth temperature and salinity stratification into a welldefined thermohaline staircase. Layers that develop initially are relatively thin and unsteady; they merge continuously.

Several recent studies, numerical and observational, have attempted to quantify the role of various layering mechanisms in the generation of oceanic staircases. Morell et al. (2006) examined field measurements of the staircase in a cyclonic eddy in the eastern Caribbean and concluded that most of the layered structures can be attributed to either intrusion or gamma instability mechanisms, possibly to a combination thereof. Stellmach et al. (2011) analyzed direct numerical simulations (DNS) of fingering convection and concluded that layering is 
consistent with the gamma instability hypothesis. In Part I of this study (Radko et al. 2014), we examined the origin and dynamics of staircases using a large-scale model of the subtropical thermocline in which fingering was parameterized rather than resolved. These simulations were consistent with the gamma instability mechanism for staircase formation.

In addition to the initiation of layering, another unresolved—and perhaps even more pressing - problem concerns the evolution of staircases and their equilibrium structure. To explain the dynamics of staircases, observers and modelers have to face a number of challenges. In most locations, staircases are in a quasiequilibrium state, and therefore the available field data do not provide a description of their full evolutionary sequence. Numerical modeling capabilities are still a long way from fully resolving all relevant spatial scales, from the diffusive salinity scale of millimeters to the equilibrium layer thickness as large as tens, in some cases hundreds, of meters. The wide range of time scales in staircase dynamics, from minutes to years, adds another element of complexity. Nevertheless, several promising attempts to explain the evolution of staircases have already been made. Direct numerical simulations (Radko 2003), initiated with uniform temperature and salinity gradients, revealed the tendency for thin layers to form first and then merge spontaneously. The solution of a one-dimensional model with parameterized double diffusion (Radko 2005) indicated that, after a series of merging events, layers attain a critical scale at which coarsening is arrested and the staircase reaches a stable equilibrium state. The three-dimensional simulations in Part I confirmed the importance of mergers as a means of controlling the average step height in equilibrated staircases. The goal of the present analysis (Part II) is to examine the dynamics of mergers in greater detail, to develop the most general mechanistic model of this phenomenon, and to quantify the merits of extant merging theories.

Double-diffusive convection comes in two distinct forms: fingering and diffusive convection. Comprehensive discussions of both phenomena are offered in Schmitt (1994) and Radko (2013). The salt-finger regime is realized when warm and salty water overlies cold and fresh-conditions that are typical for the subtropical thermocline. Diffusive convection is more common in high-latitude oceans, where temperature $T$ and salinity $S$ frequently increase downward; signatures of diffusive convection in the Arctic Ocean have been observed and recorded by Neal et al. (1969), Neshyba et al. (1971), and Padman and Dillon (1987). Our knowledge of Arctic staircases has improved dramatically since the commencement of the Ice-Tethered Profiler (ITP) Program, providing repeated sampling of the ice-covered upper ocean (Timmermans et al. 2008). While the mechanics of primary diffusive and fingering instabilities differ considerably (Turner 1973), their large-scale consequences are often dynamically similar. Thermohaline layering occurs in both types of doubly diffusive environment and, as illustrated in this study, the evolution of diffusive and fingering staircases have many features in common.

Direct numerical simulations indicate that mergers are ubiquitous in both diffusive and fingering staircases. Figure 1 presents a numerical example of the evolution of a diffusive staircase. This direct numerical simulation was initiated by a uniform background $T-S$ gradient, perturbed by small-amplitude computer-generated noise. The first stage of the experiment (Fig. 1a) is characterized by the appearance of small-scale perturbations driven by primary diffusive instabilities. Next, the irregular, vertically elongated perturbations reorganize into horizontally coherent structures (Fig. 1b) and then into well-defined layers (Fig. 1c). The subsequent evolutionary stage consists of a series of binary-merging events in which strong interfaces, characterized by large temperature and salinity jumps, grow further at the expense of weaker interfaces that gradually erode and eventually disappear. The mergers continue until there is only one interface left within the limits of our computational domain (Fig. 1f). Note that these mergers occur without any systematic vertical displacement of interfaces. This property is illustrated by tracing the location of the ultimate survivor-the interface that persists throughout the entire experiment (marked by white arrows in Figs. 1d-f). The same merging pattern was observed in the DNS of fingering staircases (Radko 2003), which suggests that the evolution and equilibrium structure of staircases can be explained in terms of a unified theoretical framework. Development and testing of such a model is one of the central objectives of this study.

The present analysis is motivated by the proposition (Radko 2005; Part I) that mergers exert a controlling influence on the geometry of thermohaline staircases. In this scenario, the point when mergers cease sets the equilibrium step height in staircases and, consequently, determines the vertical transport of heat and salt. Several attempts have already been made to explain the mechanisms of layer merging in thermohaline staircases (Huppert 1971; Kelley 1988; Radko 2003, 2005). One of the uncertainties in the formulation of these models concerns their reliance on the four-thirds flux laws (Turner 1965, 1967) that are used to represent heat and salt transport through high-gradient interfaces. The application of the four-thirds flux laws to thermohaline staircases has been repeatedly questioned (Kunze 1987; Kelley et al. 2003). Therefore, the proposed model 


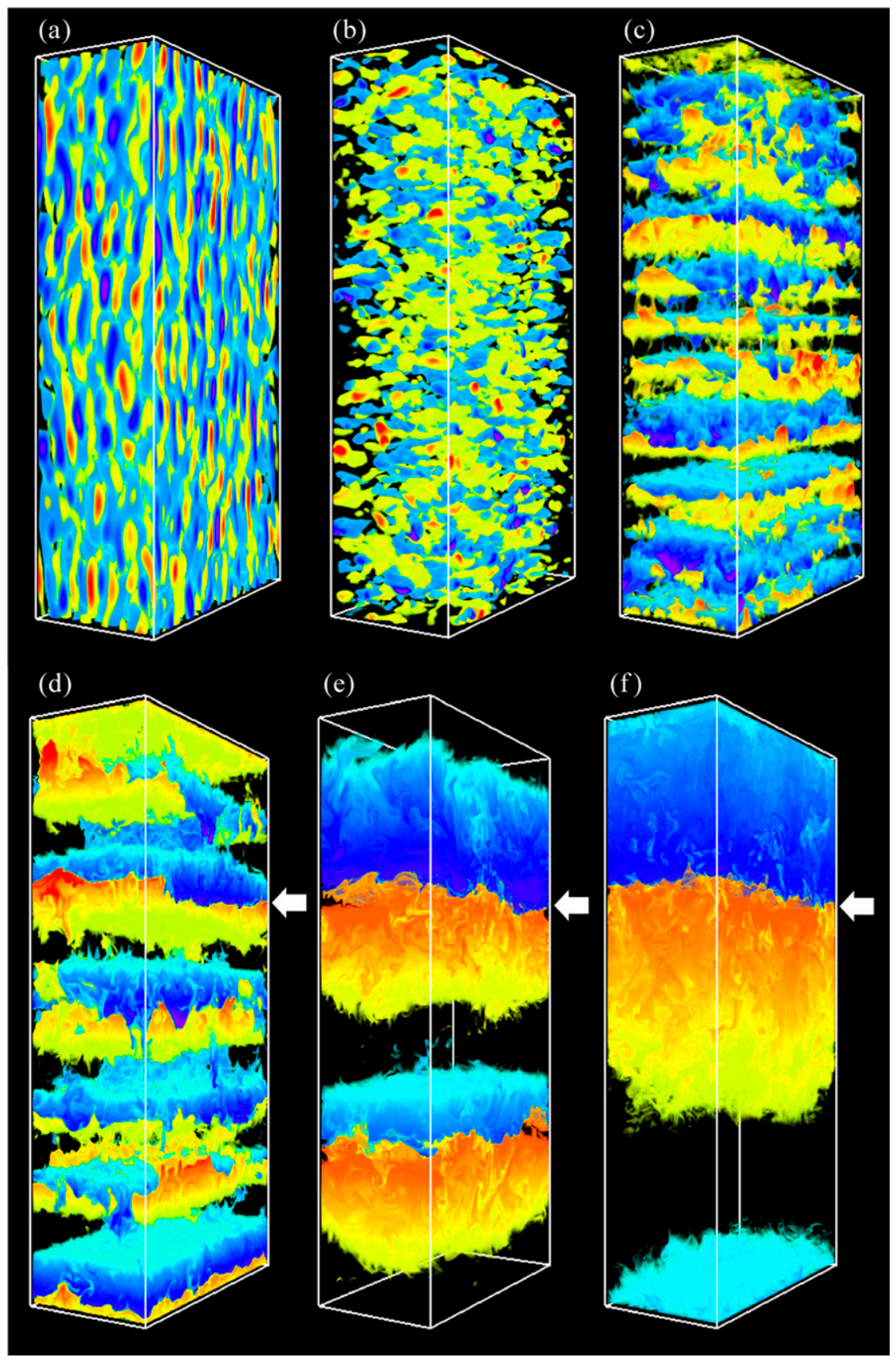

FIG. 1. Formation and evolution of layers in a DNS of diffusive convection. The experiment was initialized by a uniform, diffusively favorable gradient. Presented are the instantaneous deviations of temperature fields from the uniform background gradient at time $t$ equal to (a) 89 , (b) 166, (c) 283, (d) 298, (e) 325, and (f) $337 \mathrm{~h}$. Red (blue) corresponds to high (low) values of temperature. The white arrows in (d)-(f) point to the interface that persists through a series of mergers. The background density ratio is $R_{\rho}^{*}=1.1$, the diffusivity ratio is $\tau=k_{S} / k_{T}=1 / 6$, and the size of the computational domain is $3.4 \mathrm{~m} \times 1.7 \mathrm{~m} \times 6.9 \mathrm{~m}$, which is resolved by $768 \times 384 \times 1536$ grid points. Detailed description of the numerical model is given in section 3 . 
(a)

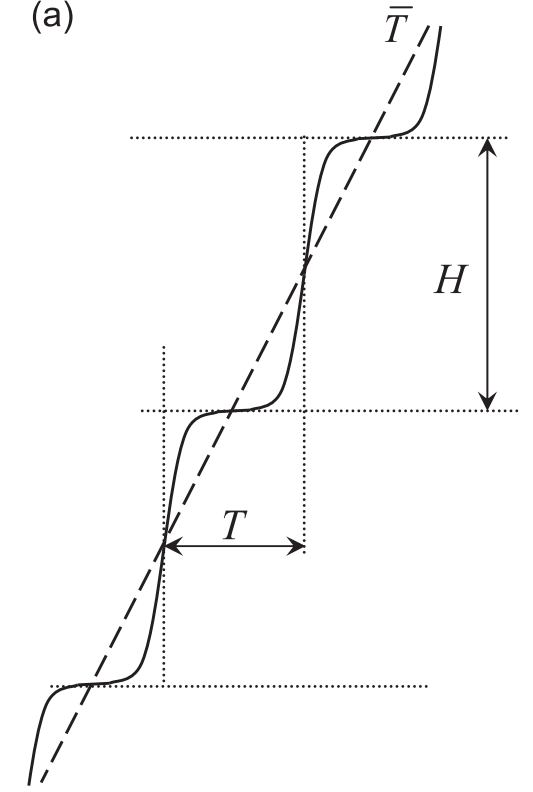

(b)

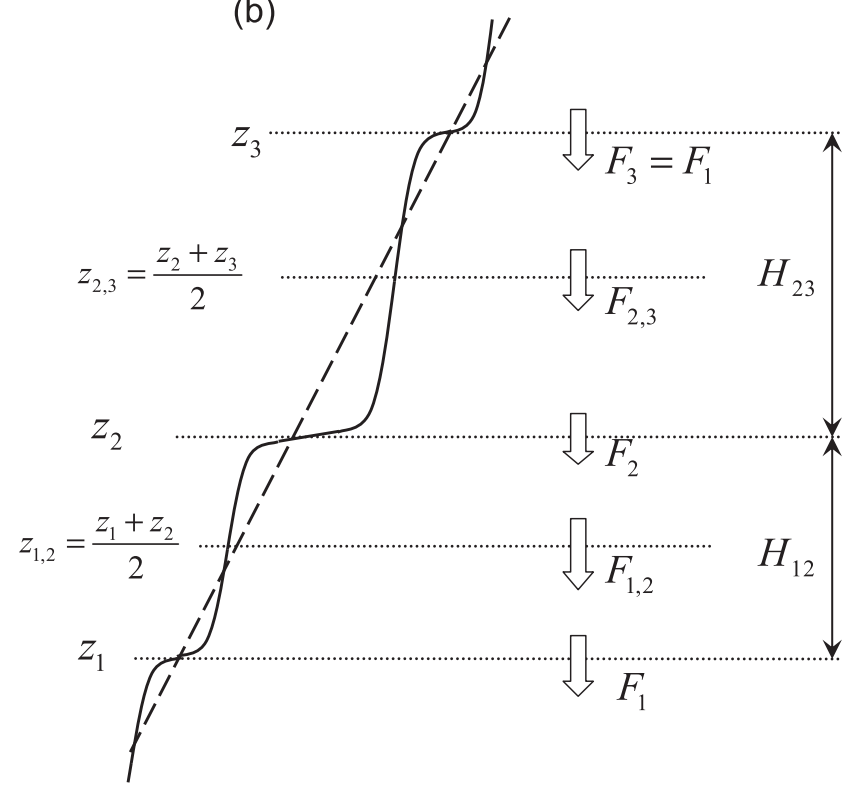

FIG. 2. Schematic diagram [after Radko (2007)] illustrating the stability analysis for an infinite series of layers and interfaces. (a) Basic state consisting of identical steps. (b) Perturbed state in which the $T-S$ jumps at even and odd interfaces are modified in a compensating manner that does not affect the background large-scale gradients $\left(\bar{T}_{z}, \bar{S}_{z}\right)$.

attempts to describe merging events in a less restrictive manner, without assuming from the outset specific relations for the vertical fluxes of heat and salt. We find that merging models based on the four-thirds flux laws offer adequate qualitative description of the evolutionary patterns. However, they are less accurate than our new model that does not rely on such laws. In this study, merging events are also examined through the analysis of field data. The inspection of several instances of mergers in the Arctic ITP data reveals that their patterns generally conform to the prediction of the layer-merging model.

This paper is organized as follows. Section 2 presents the layer-merging staircase theory in its most general form, without assuming specific flux laws. The model is applicable for both diffusive and fingering staircases. In section 3, we examine a series of direct numerical simulations, which validate and quantify the analytical arguments. Next, we identify merging events in the ITP data for the Arctic staircases and interpret them in terms of the proposed merging theory (section 4). We summarize and draw conclusions in section 5 .

\section{Theory}

As is common in theoretical discussions of staircase evolution (Huppert 1971; Radko 2005, 2007), layermerging events are best described in terms of the stability problem illustrated in Fig. 2. We consider the basic steady state consisting of identical layers of thickness $H$
(Fig. 2a) that are perturbed in the manner indicated in Fig. 2b. Variations in temperature, salinity, and buoyancy across the interfaces are slightly modified relative to that in the basic state, and the interfaces are slightly displaced vertically. The system in Fig. 2 is periodic with the $z$ wavelength of $2 \mathrm{H}$, and perturbing the basic state in such a manner does not affect the background $T-S$ gradients. Our objective is to determine the susceptibility of this system to spontaneous layer-merging events.

Radko (2007) suggested that layered systems can exhibit two distinct modes of instability: $\mathrm{B}$ and $\mathrm{H}$ instabilities. In the first mode (B instability), slightly stronger interfaces strengthen further at the expense of weaker interfaces, which gradually erode as indicated in Fig. 3a. In the second mode ( $\mathrm{H}$ instability), slightly thicker layers thicken even more, while thin layers shrink and ultimately disappear (Fig. 3b). These instabilities are controlled by the dependencies of the heat/salt fluxes in each step $\left(\tilde{F}^{T}, \tilde{F}^{S}\right)$ on its temperature and salinity variations $(\tilde{T}, \tilde{S})$ and the step height $(\tilde{H})$ :

$$
\left\{\begin{array}{l}
\tilde{F}^{T}=\tilde{F}^{T}(\tilde{T}, \tilde{S}, \tilde{H}) \quad \text { and } \\
\tilde{F}^{S}=\tilde{F}^{S}(\tilde{T}, \tilde{S}, \tilde{H}) .
\end{array}\right.
$$

In our analysis (details of which are given in the appendix), the interfacial variations of properties $(\tilde{T}, \tilde{S})$ are defined as differences in temperature and salinity between the centers of the layer immediately above the interface and the layer below. Cross-interfacial fluxes 

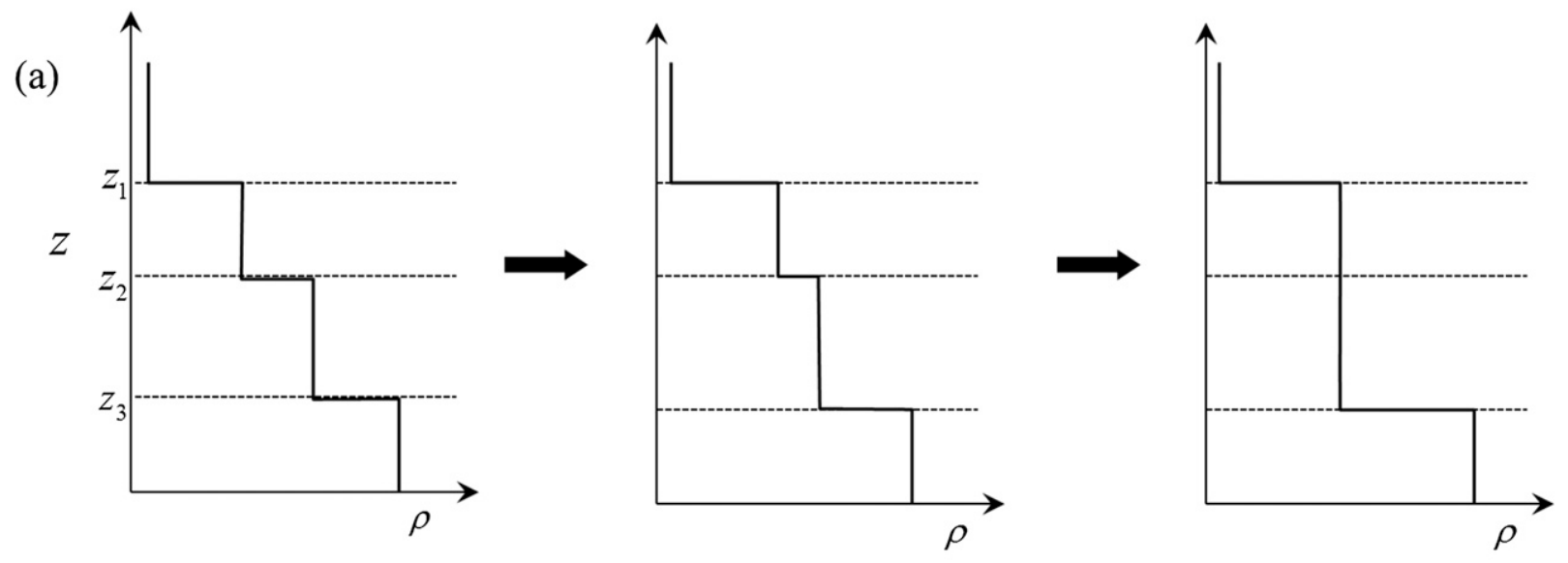

(b)
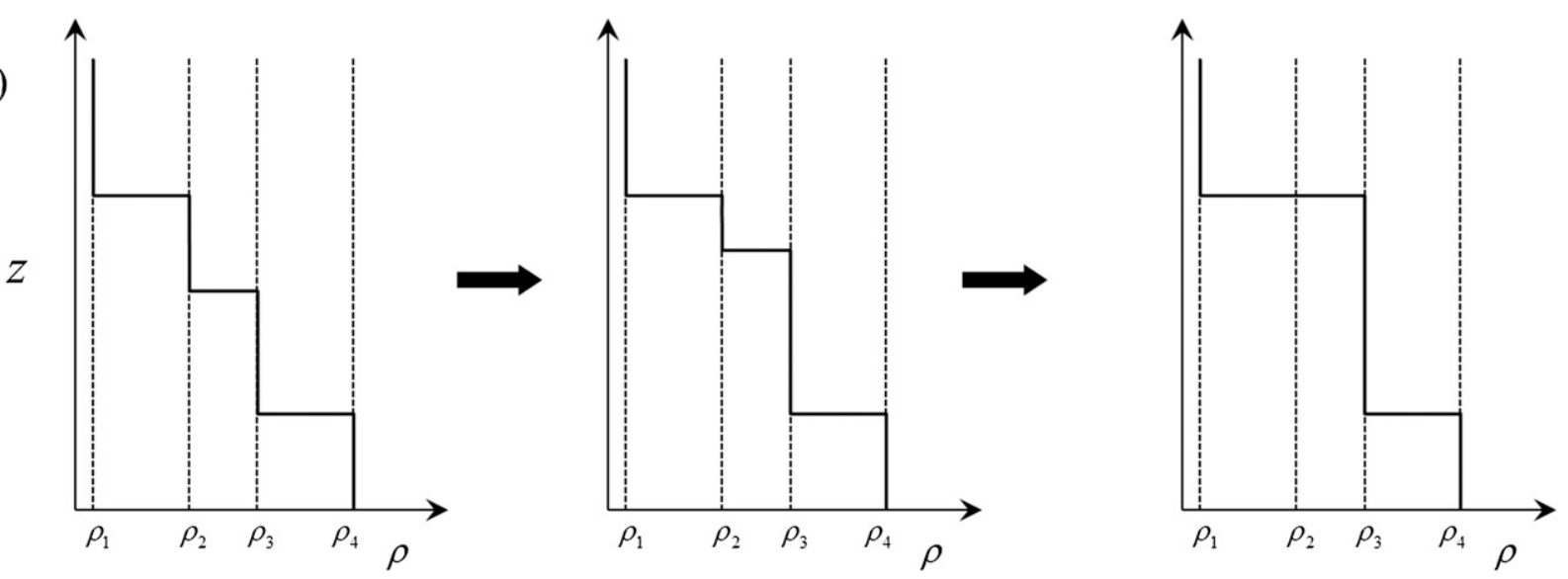

FIG. 3. Schematic representation of the two possible merging scenarios: (a) the B merger, which occurs when some interfaces gradually erode without moving vertically, and (b) the $\mathrm{H}$ merger, which occurs when interfaces drift vertically and collide. Numerical simulations and field data indicate that B mergers are more common in thermohaline staircases.

$\left(\tilde{F}^{T}, \tilde{F}^{S}\right)$ are defined positive downward (as indicated in Fig. 2), in the salt-finger sense. However, the chosen convention does not lead to any loss of generality, and the theoretical framework is fully applicable to diffusive systems as well.

The extant merging models (Radko 2005, 2007) suggest that the variation of fluxes with $\tilde{T}$ and $\tilde{S}$ can lead to B mergers, whereas the variation of fluxes with $\tilde{H}$ results in $\mathrm{H}$ mergers. If both modes of instability are engaged, the preferred evolutionary pattern is determined by the unstable mode with the largest growth rate. In the context of the thermohaline staircase problem, much more common is the B-merger scenario. Merging by the erosion of weak interfaces has been observed in all our numerical simulations (e.g., Fig. 1), as well as in the ITP data (section 5). An example of the B merger in field data, discussed in Part I, was presented by Zodiatis and Gasparini (1996), who documented temporal changes in the Tyrrhenian Sea staircase. However, it should be noted that $\mathrm{H}$ mergers have been observed in some laboratory experiments with one-component fluids (e.g., Ruddick et al. 1989; Park et al. 1994). The reason for the general preference for the B-merger events can be rationalized using the same classical argument that is invoked in the justification of Turner's four-thirds laws. The vertical $T-S$ fluxes are assumed to be controlled by the high-gradient interfaces, which play the role of transport bottlenecks for the mixing of heat and salt in thermohaline staircases. The interfaces respond to conditions in their immediate vicinity. Thus, if layers are sufficiently thick, fluxes across each interface are not sensitive to the step heights and are determined by the variation of temperature and salinity across each step. In this case, the staircase is expected to evolve in time following the B-merger scenario. The $\mathrm{H}$ mergers can occur at the initial stages of the staircase coarsening process, where interfaces are still affected by the circulation at the adjacent steps, but not in mature oceanic staircases with thick fully developed mixed layers.

Given the overall preference for B-type mergers, we focus our analysis on the corresponding instability mode. 
In the appendix, we derive an expression for the B-instability growth rate for the configuration in Fig. 2:

$$
\begin{aligned}
\lambda^{2} & +\frac{4}{H}\left(\frac{\partial \tilde{F}^{T}}{\partial \tilde{T}}+\frac{\partial \tilde{F}^{S}}{\partial \tilde{S}}\right) \lambda \\
& +\frac{16}{H^{2}}\left(\frac{\partial \tilde{F}^{T}}{\partial \tilde{T}} \frac{\partial \tilde{F}^{S}}{\partial \tilde{S}}-\frac{\partial \tilde{F}^{S}}{\partial \tilde{T}} \frac{\partial \tilde{F}^{T}}{\partial \tilde{S}}\right)=0 .
\end{aligned}
$$

This expression can be made explicit if the free coefficient is small, which is to be expected on the basis of simple scaling arguments (see the appendix). In this case, the larger-positive in the case of instability—root can be approximated by

$$
\lambda=\frac{4}{H} \frac{\frac{\partial \tilde{F}^{S}}{\partial \tilde{T}} \frac{\partial \tilde{F}^{T}}{\partial \tilde{S}}-\frac{\partial \tilde{F}^{T}}{\partial \tilde{T}} \frac{\partial \tilde{F}^{S}}{\partial \tilde{S}}}{\frac{\partial \tilde{F}^{T}}{\partial \tilde{T}}+\frac{\partial \tilde{F}^{S}}{\partial \tilde{S}}} .
$$

The conceptual significance of the relationships (2) and (3) lies in their generality. Previous evolutionary models of thermohaline staircases were based on the four-thirds flux laws (Turner 1965, 1967):

$$
\left\{\begin{array}{l}
\alpha \tilde{F}^{T}=C\left(R_{\rho}\right)(\alpha \tilde{T})^{4 / 3} \text { and } \\
\beta \tilde{F}^{S}=\frac{\alpha \tilde{F}^{T}}{\gamma\left(R_{\rho}\right)}
\end{array}\right.
$$

where $C$ is the flux-law coefficient, $\gamma$ is the flux ratio, and $(\alpha, \beta)$ are expansion/contraction coefficients of the assumed linear equation of state. Both $C$ and $\gamma$ are assumed to be determined by the density ratio $R_{\rho}=\alpha \tilde{T} / \beta \tilde{S}$. While (4) finds support in laboratory experiments (e.g., Schmitt 1979a), serious doubts have been raised (Kunze 2003; Kelley et al. 2003) with regard to the relevance of the four-thirds laws for oceanic staircases. Kunze (1987) finds that the extrapolation of the laboratory-calibrated fourthirds flux laws in the salt-finger regime overestimates the observed fluxes in the Caribbean staircase by more than an order of magnitude.

While the situation with regard to diffusive staircases may not be as extreme, the diffusive flux laws should also be applied to observations with caution. For instance, Flanagan et al. (2013) performed a series of direct numerical simulations in the parameter regime relevant for the Arctic staircase. These simulations suggest that extant laboratory-derived flux laws (e.g., Marmorino and Caldwell 1976; Kelley 1990) can underestimate heat transport by as much as a factor of 2-3 and even the fluxlaw exponent may require downward revision from 1.33 to 1.11. Flanagan et al. (2013) attribute the discrepancy between the laboratory-derived and numerical results to the presence of rigid boundaries and the rundown character of the laboratory experiments. The uncertainties with regard to the four-thirds flux laws could be exacerbated in the context of the layer-merging problem. Mergers are driven by subtle imbalances of $T-S$ fluxes in the adjacent steps, and therefore even modest inaccuracies in the formulation of the flux laws may lead to substantial errors in predicting the evolutionary patterns of staircases.

On the other hand, it is comforting to see that if the four-thirds laws are assumed, the general result (3) reduces to earlier estimates of merging growth rate. Thus, substituting (4) into the growth rate expression (3), we arrive at

$$
\lambda=\frac{(\alpha \tilde{T})^{1 / 3}}{H} G\left(R_{\rho}\right)
$$

where

$$
G\left(R_{\rho}\right)=\frac{16 C^{2} \frac{\partial \gamma}{\partial R_{\rho}} R_{\rho}^{2}}{3 \frac{\partial C}{\partial R_{\rho}} \gamma R_{\rho}^{2}-4 C \gamma^{2}-3 \frac{\partial C}{\partial R_{\rho}} \gamma^{2} R_{\rho}-3 C \frac{\partial \gamma}{\partial R_{\rho}} R_{\rho}^{2}}
$$

The expressions (5) and (6) are fully consistent with previous layer-merging models (Huppert 1971; Radko 2005). In particular, the sign of the growth rate-hence the stability/instability of a staircase-is controlled by the flux ratio pattern. If the flux ratio decreases with increasing density ratio $\left(\partial \gamma / \partial R_{\rho}<0\right)$, then the system of layers is unstable and layers tend to merge spontaneously. Otherwise, the staircase is fully equilibrated. Laboratory experiments (e.g., Turner 1965; Schmitt 1979a) and direct numerical simulations (e.g., Traxler et al. 2011; Radko and Smith 2012) indicate that, in both fingering and diffusive regimes, the gamma instability condition $\partial \gamma / \partial R_{\rho}<0$ is satisfied when the density ratio is sufficiently close to unity. However, as layers merge, the system evolves toward a more stable configuration, and merging events eventually cease (Radko 2005).

\section{Numerics}

\section{a. Formulation}

To test the foregoing layer-merging theory and, in particular, to assess the merits of the four-thirds flux laws, we turn to direct numerical simulations. The temperature and salinity fields are separated into the basic state $(\bar{T}, \bar{S})$, representing a uniform vertical gradient and a departure 
TABLE 1. The domain and grid sizes for the merging experiments (Expt) 1-4 are listed along with the interfacial density ratios pre- and postmerger.

\begin{tabular}{lcccc}
\hline \hline & & & \multicolumn{2}{c}{ Interfacial density ratio } \\
\cline { 3 - 5 } & \multicolumn{1}{c}{ Size } & Grid & Premerger & 1.3345 \\
SF2D (Expt 1) & $400 \times 400$ & $6144 \times 6144$ & 3.785 & 1.4745 \\
DC2D (Expt 2) & $60 \times 60$ & $1536 \times 1536$ & 1.118 & 4.435 \\
SF3D (Expt 3) & $335 \times 335 \times 536$ & $384 \times 384 \times 768$ & 1.383 & 1.129 \\
DC3D (Expt 4) & $90 \times 45 \times 180$ & $192 \times 96 \times 768$ & 1.435 \\
\hline
\end{tabular}

$(T, S)$ from it. The governing Boussinesq equations of motion are expressed in terms of perturbations $T$ and $S$. To reduce the number of controlling parameters, the system is nondimensionalized using $l=\left(k_{T} \nu / g \alpha\left|\bar{T}_{z}\right|\right)^{1 / 4}$, $k_{T} / l, l^{2} / k_{T}$, and $\rho_{0} \nu k_{T} / l^{2}$ as the scales of length, velocity, time, and pressure, respectively. Here, $\left(k_{T}, k_{S}\right)$ denote the molecular diffusivities of heat and salt, and $\rho_{0}$ is the reference density used in the Boussinesq approximation. The expansion/contraction coefficients $(\alpha, \beta)$ are incorporated in $(T, S)$, and $\alpha \bar{T}_{z} l$ is used as the scale for both temperature and salinity perturbations. To distinguish between nondimensional and dimensional variables, the latter will be denoted in the remainder of this section by the subscript "dim."

For the fingering regime $\left(\bar{T}_{z}>0, \bar{S}_{z}>0\right)$, the governing equations reduce to

$$
\left\{\begin{array}{l}
\frac{\partial T}{\partial t}+\mathbf{v} \cdot \nabla T+w=\nabla^{2} T \\
\frac{\partial S}{\partial t}+\mathbf{v} \cdot \nabla S+\frac{w}{\bar{R}_{\rho}}=\tau \nabla^{2} S \\
\frac{1}{\operatorname{Pr}}\left(\frac{\partial}{\partial t} \mathbf{v}+\mathbf{v} \cdot \nabla \mathbf{v}\right)=-\nabla p+(T-S) \mathbf{k}+\nabla^{2} \mathbf{v}, \text { and } \\
\nabla \cdot \mathbf{v}=0
\end{array}\right.
$$

where $\bar{R}_{\rho}=\alpha \bar{T}_{z_{\mathrm{dim}}} / \beta \bar{S}_{z_{\mathrm{dim}}}$ is the background density ratio, $\tau=k_{S} / k_{T}$ is the diffusivity ratio, $\operatorname{Pr}=\nu / k_{T}$ is the Prandtl number, $\mathbf{v}=(u, v, w)$ is the velocity vector, and $\mathbf{k}$ is the vertical unit vector. The system used for diffusive convection $\left(\bar{T}_{z}<0, \bar{S}_{z}<0\right)$ is identical to (7) in all respects except for the sign of the $w$ terms in the temperature and salinity equations, which take the following form:

$$
\left\{\begin{array}{l}
\frac{\partial T}{\partial t}+\mathbf{v} \cdot \nabla T-w=\nabla^{2} T \quad \text { and } \\
\frac{\partial S}{\partial t}+\mathbf{v} \cdot \nabla S-\bar{R}_{\rho}^{*} w=\tau \nabla^{2} S .
\end{array}\right.
$$

In discussions of diffusive convection, it is more common to use the diffusive density ratio $\bar{R}_{\rho}^{*}=1 / \bar{R}_{\rho}=\beta \bar{S}_{z_{\text {dim }}} / \alpha \bar{T}_{z_{\text {dim }}}$ and the diffusive flux ratio $\gamma^{*}=\beta \tilde{F}_{\mathrm{dim}}^{S} / \alpha \tilde{F}_{\mathrm{dim}}^{T}$ in lieu of $R_{\rho}$ and $\gamma$. We shall follow suit.

DNS of double-diffusive convection, both fingering and diffusive, still represent a considerable computational challenge, particularly in the oceanographic (heatsalt) context. The key difficulty is the vast separation of spatial and temporal scales that require adequate resolution in the staircase problem. The scale of salt dissipation is less than the scale of heat dissipation by a factor of $\sqrt{\tau} \ll 1$, and the scale of heat dissipation is, in turn, much less than the typical step height in the staircase. Merging simulations are especially demanding because the time scale of merging events-typically several weeks or more-greatly exceeds that of primary double-diffusive effects (measured in minutes). Such severe computational constraints place three-dimensional merging DNS for the heat-salt parameters $(\tau \sim 0.01)$ beyond the reach of modern computers. Staircase modelers have two options: either to compromise on the governing parameters and use the diffusivity ratio that is higher than the heatsalt value or to resort to two-dimensional simulations. On the positive side, neither approach leads to qualitative inconsistencies. As discussed in Stern et al. (2001) and Radko (2008), the use of a moderate diffusivity ratio is not expected to alter the fundamental physics and characteristics of salt fingering, as long as $\tau$ remains significantly less than unity. Three-dimensional simulations tend to elevate vertical fluxes by a factor of 2 or so relative to the corresponding 2D simulations. However, on the qualitative level, all major double-diffusive phenomena appear to be adequately represented by two-dimensional models. It should also be noted that sheared environments favor formation of salt sheets aligned in the direction of the background current (Linden 1974; Kimura and Smyth 2007), in which case double-diffusive dynamics become effectively two-dimensional. It is perhaps ironic that because large-scale shears are ubiquitous in the ocean, salt fingers may be better represented by twothan by three-dimensional simulations.

In this study, we pursue both approaches. We use twodimensional simulations to focus directly on the oceanographically relevant parameters $(\tau \sim 0.01)$. To ensure that these calculations are not compromised by neglecting 
(a)

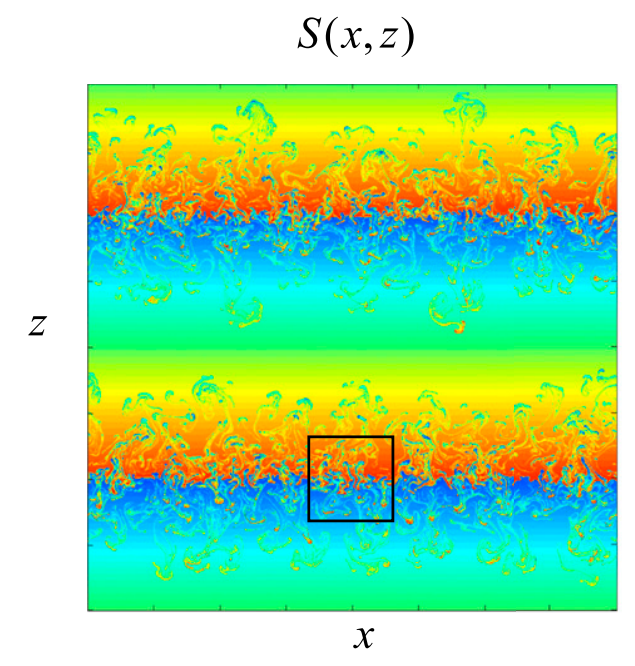

(b)

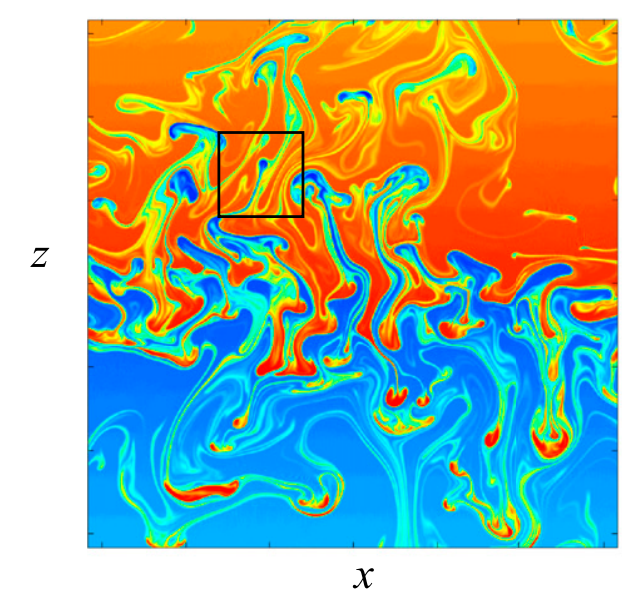

(c)

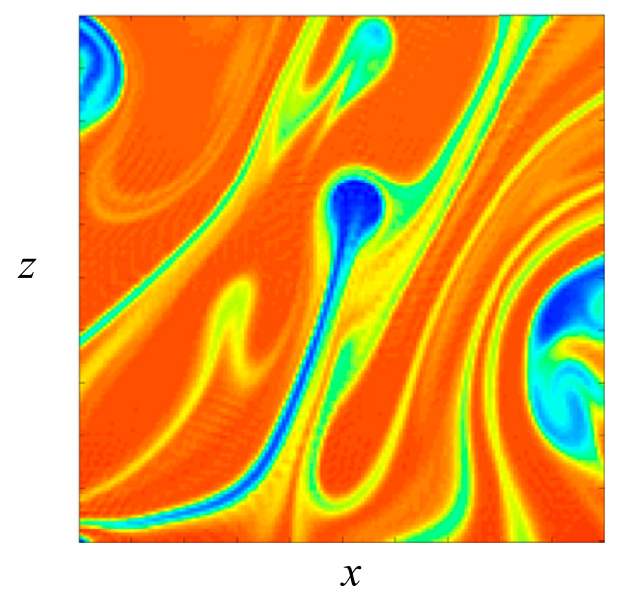

FIG. 4. Instantaneous salinity field for the numerical experiment 1 (two-dimensional fingering). (a) The whole computational domain; (b) an enlarged view of the square area marked in (a); and (c) an enlarged view of the square area in (b). The domain size in this calculation is $\left(L_{x}, L_{z}\right)=(400,400)$ and the numerical mesh consists of $\left(N_{x}, N_{z}\right)=(6144,6144)$ nodes. The sizes of the regions shown in (b) and (c) are $63 \times 63$ and $10 \times 10$ nondimensional units, respectively. fundamentally three-dimensional effects, we supplement our two-dimensional simulations by three-dimensional runs in a computationally accessible regime. Dynamical consistency of the two- and three-dimensional simulations instills greater confidence in the ability of our models to capture the zero-order dynamics at play. The discussion in this section is based on the analysis of four representative merging experiments:

- Experiment 1: 2D fingering simulation with realistic parameters $\left(\bar{R}_{\rho}, \tau, \operatorname{Pr}\right)=(1.3,0.01,7)$;

- Experiment 2: $2 \mathrm{D}$ diffusive simulation with realistic parameters $\left(\bar{R}_{\rho}^{*}, \tau, \operatorname{Pr}\right)=(3,0.005,13)$;

- Experiment 3: 3D fingering simulation in the accessible regime $\left(\bar{R}_{\rho}, \tau, \operatorname{Pr}\right)=(1.1,1 / 3,7)$; and

- Experiment 4: 3D diffusive simulation in the accessible regime $\left(\bar{R}_{\rho}^{*}, \tau, \operatorname{Pr}\right)=(1.3,1 / 16,13)$.

Experiment 1 represents relatively mild tropical/ midlatitude conditions, characteristic of fingering staircases in the Caribbean, Tyrrhenian, and Mediterranean, where $\tau \sim 0.01, \operatorname{Pr} \approx 7$, and $1.1<R_{\rho}<1.7$. Experiment 2 was designed to represent diffusive staircases in the Beaufort Gyre of the Arctic Ocean. The diffusivity ratio in cold Arctic waters $(\tau \sim 0.005)$ is less than in typical fingering staircases (experiment 1). The high-latitude Prandtl number $(\operatorname{Pr}=13)$ is larger than in experiment 1 , and the representative diffusive density ratio is $\bar{R}_{\rho}^{*} \sim$ 3 . The choice of the diffusivity ratio in experiments 3 and 4 was dictated by considerations of computational feasibility. In each 3D experiment, the best resolution was used, given the available resources. The diffusivity ratio $\tau$, in turn, was taken to be as small as possible while still adequately resolving the salinity dissipation scale. The result of this increase in $\tau$ is a change in the pattern of the flux ratio, which shifts the merger-favorable parameter range toward the density ratios close to unity. Therefore, the density ratios used in three-dimensional simulations are also unrealistically low: $\bar{R}_{\rho}=1.1$ for the fingering experiment 3 and $\bar{R}_{\rho}^{*}=1.3$ for the diffusive experiment 4 . The domain and grid sizes used for each experiment are listed in Table 1.

Each of these experiments was initiated by the state at rest with the $T-S$ distribution consisting of two identical steps. The initial steps were represented by perfectly mixed layers with homogeneous total temperature and salinity patterns, separated by horizontal discontinuous interfaces. To expedite development of double-diffusive instabilities, the initial $T-S$ distribution was slightly perturbed by small-amplitude, random, computer-generated noise. We assumed periodic boundary conditions for $(T, S, \mathbf{v}, p)$ in each spatial direction and integrated the governing equations using the dealiased pseudospectral method, described and used in numerous 

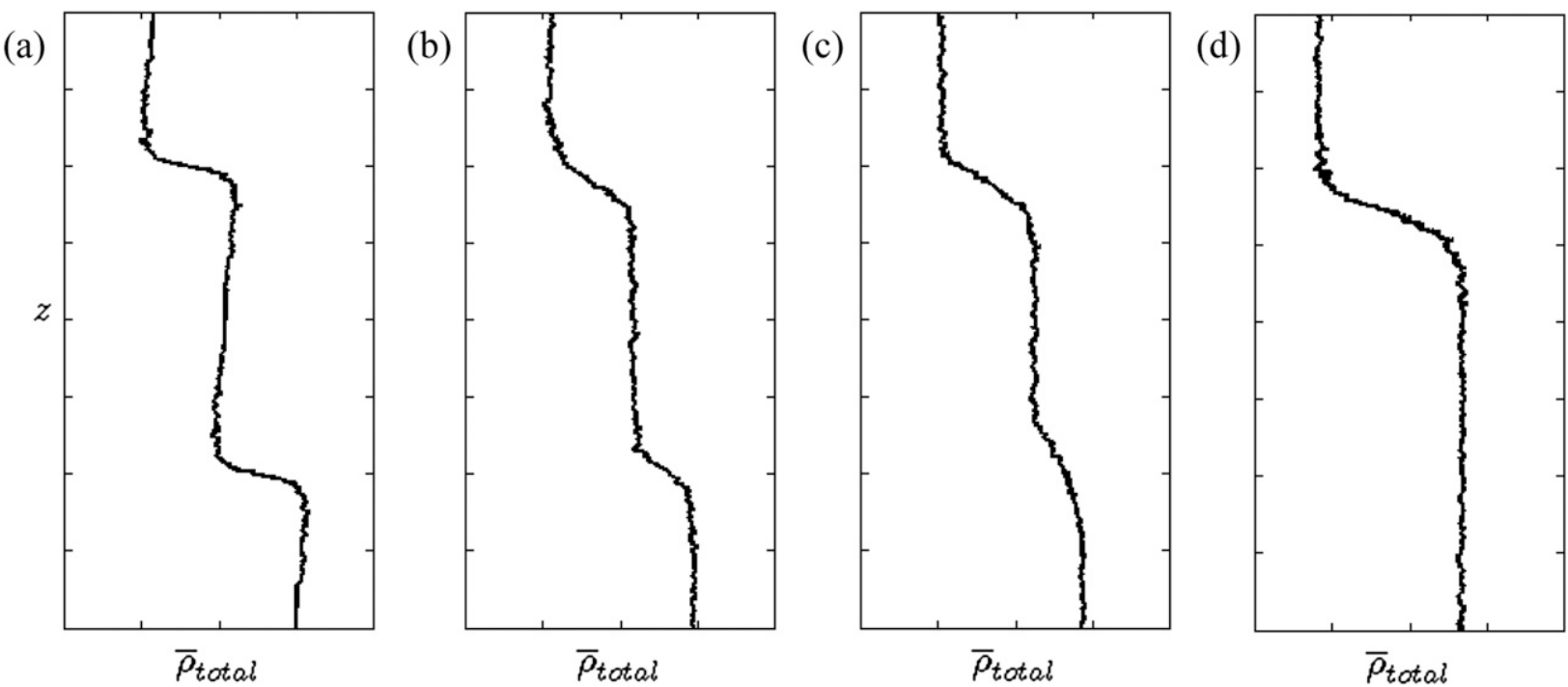

FIG. 5. The total $x$-averaged density for the experiment 1 at $t$ equal to (a) 5, (b) 459, (c) 543, and (d) 736. Note the gradual erosion of the weaker (lower) interface and its eventual disappearance, suggestive of the B-merger dynamics.

double-diffusive modeling studies (e.g., Stern et al. 2001; Traxler et al. 2011; Stellmach et al. 2011). The model employed adaptive time stepping; at each time level, the optimal step $(\Delta t)$ was chosen internally in a way that ensures numerical stability of the code.

\section{b. Results}

Figure 4 presents the salinity distribution at the early stage of experiment 1 at $t=6$ (equivalent to the dimensional value of $t_{\mathrm{dim}} \approx 1 \mathrm{~h}$ ). The domain size in this calculation $\left(L_{x}, L_{z}\right)=(400,400)$ corresponds to the dimensional values of $\left(L_{x_{\mathrm{dim}}}, L_{z_{\mathrm{dim}}}\right)=(3.7 \mathrm{~m}, 3.7 \mathrm{~m})$. Figure 4 vividly illustrates the wide range of dynamically active scales in the staircase problem and the associated numerical complications. The entire computational domain is shown in Fig. 4a, while Fig. 4b presents an enlarged view of the small square area marked in Fig. 4a. Figure 4c, in turn, zooms in on a small area in Fig. 4b and shows one of the salt fingers forming at the high-gradient interface. The evolutionary pattern of the two-layer system is revealed in Figs. 5a-d, which presents the total horizontally averaged density field $\bar{\rho}_{\text {total }}$ at various times. It shows the gradual merger of two initially identical layers into a single step that spans the entire computational domain. The merger pattern suggests that the evolution of the system follows the B-merger scenariocontinuous strengthening of the stronger interface at the expense of the weaker one, which gradually erodes and eventually disappears.

The simulation in Fig. 6 represents the evolution of the diffusive system (experiment 2), which proved to be analogous to that in experiment 1 . Two nearly identical layers gradually merge as the property variations across the slightly stronger interface monotonically increase, while the variations across the weaker interface decrease until the interface vanishes completely. The horizontally averaged density profiles in Fig. 7 reveal that interfaces remain largely confined to their initial positions throughout the experiment, in accord with the B-merger scenario.

Figure 8 attempts to quantify the strength of the merging perturbation in the experiments shown in Figs. 4-7 and examine its temporal pattern. Here, we plot the difference in the temperature jumps across the two interfaces:

$$
\Delta T_{s w}=\tilde{T}_{s}-\tilde{T}_{w}
$$

where $\tilde{T}_{s}\left(\tilde{T}_{w}\right)$ denotes the temperature jump across the stronger (weaker) interface. This difference gradually increases in time to the maximum value of $\bar{T}_{z} L_{z}$ following the complete merger of two steps. Of particular significance is the exponential character of $\Delta T_{s w}(t)$, which confirms that the merging process is appropriately interpreted (section 2) as a manifestation of staircase instability. The exponential growth of merging perturbation is illustrated by superimposing the numerical record of $\Delta T_{s w}(t)$ with its exponential fit:

$$
\Delta T_{\text {fit }}(t)=\Delta T_{\text {fit }}(0)+\hat{T}\left[\exp \left(\lambda_{\text {num }} t\right)-1\right]
$$

The analysis based on the temporal variation of salinity, rather than temperature, reveals similar exponential growth 
(a)

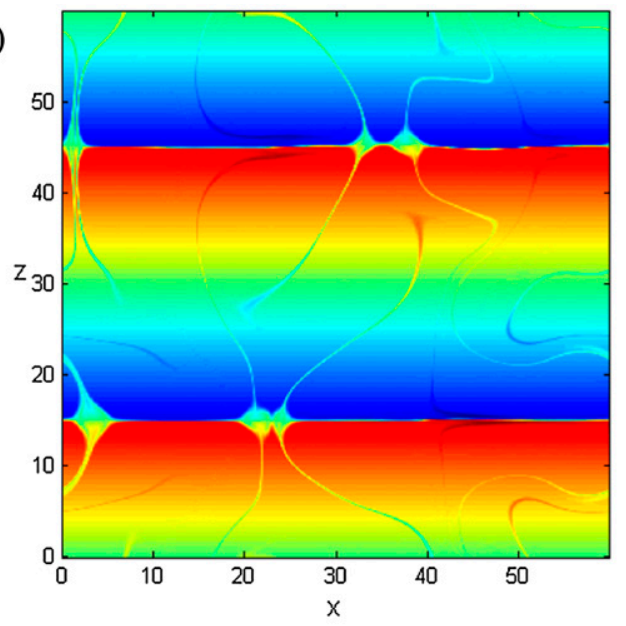

(c)

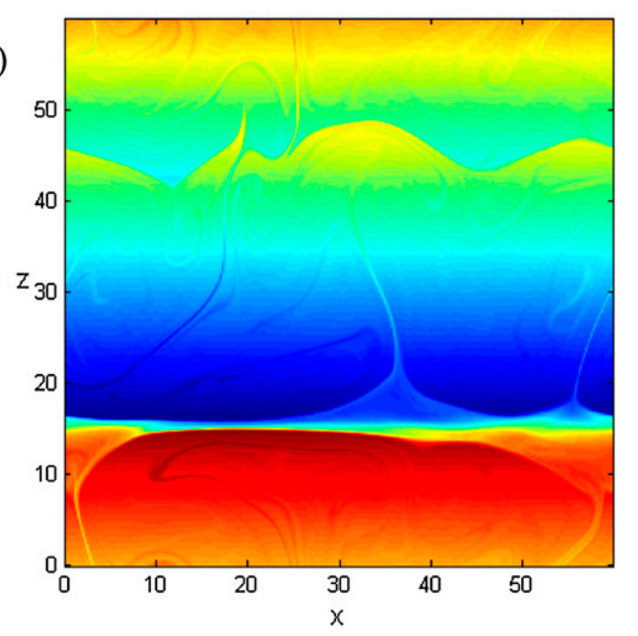

(b)

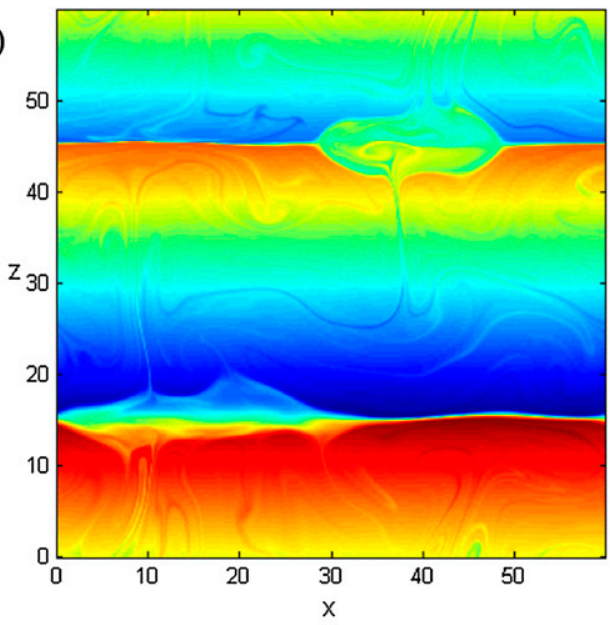

(d)

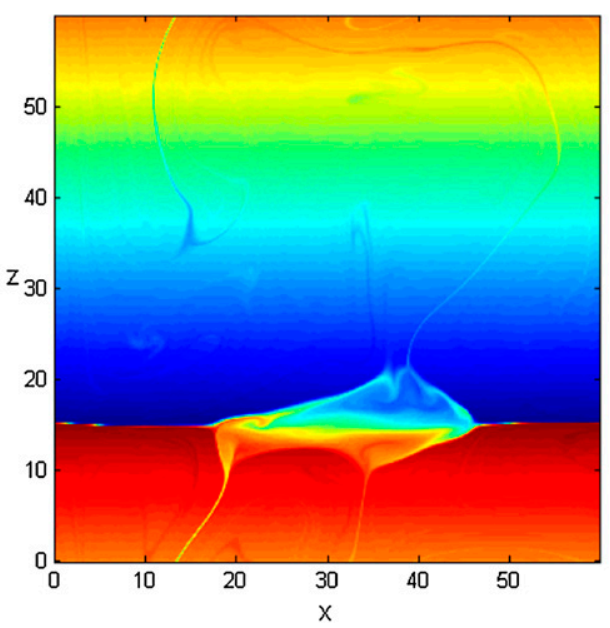

FIG. 6. Evolution of the perturbation salinity field $S$ for experiment 2 (two-dimensional diffusive convection). The upper interface gradually weakens and eventually disappears. Salinity patterns are shown at $t$ equal to (a) 9 , (b) 20651 , (c) 26462, and (d) 26807.

of merging perturbations and yields nearly identical estimates of its growth rate.

Aside from their high-frequency variability, which is expected for turbulent double-diffusive processes, the time records of merging perturbations closely follow their exponential fits during the linear ( $\operatorname{small} \Delta T_{s w}$ ) simulation phase. This is a generic property of merging simulations, realized both in fingering (e.g., Fig. 8a) and diffusive (e.g., Fig. 8b) experiments. It is also interesting to examine the final fully nonlinear phase of the experiments, which is characterized by the rapid acceleration of mergers. The difference in the linear and nonlinear merging rates is particularly dramatic for the diffusive experiments. Thus, for the simulation in Fig. 8b, the temperature perturbation exponentially increased over the period $0<t<2.4 \times 10^{4}$ (equivalent to the dimensional time of 6 months) during which the temperature perturbation reached $\Delta T_{s w} \approx 20$. Upon reaching this amplitude, perturbation growth temporarily slowed $\left(2.4 \times 10^{4}<t<\right.$ $\left.2.65 \times 10^{4}\right)$ and then rapidly increased to $\Delta T_{s w} \approx 60$ over a time interval of $\Delta t \approx 500$, corresponding to the dimensional time scale of only 4 days. The difference in time scales between the protracted preconditioning phase and the rapid final stage becomes important in the discussion of mergers observed in the Arctic staircases (section 4).

The evolutionary features seen in two-dimensional experiments (Figs. 4-8) have also been realized in threedimensional simulations (experiments 3 and 4). In Fig. 9, we present visualizations of the temperature perturbation fields shortly after the beginning of each run and at their final stages. Recall that in order to make 3D simulations feasible, the diffusivity and density ratios have been modified relative to their typical oceanic values. Nevertheless, the three-dimensional simulations in Fig. 9, both fingering (Fig. 9a) and diffusive (Fig. 9b), are similar 

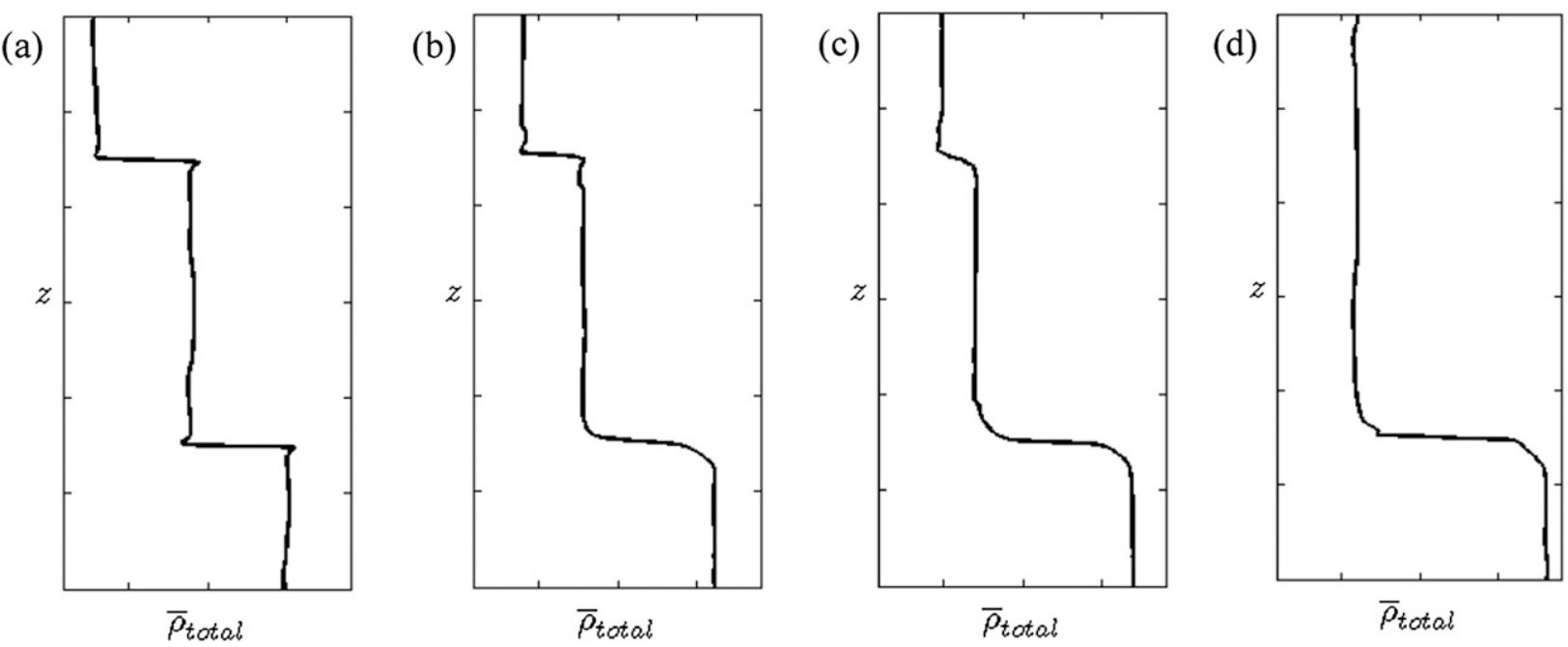

FIG. 7. As in Fig. 5, but for experiment 2. Density profiles are shown at $t$ equal to (a) 9, (b) 20 651, (c) 23393 , and (d) 26807.

to their two-dimensional counterparts. They exhibit all the defining characteristics of the B merger: the absence of vertical interfacial drift and the gradual erosion of weaker interfaces. It is interesting that the preference for B mergers seems to be an inherently double-diffusive effect. For instance, Radko (2007) considered the evolution of layers in a one-component turbulent fluid. The parametric 1D model of mechanical turbulence produced $\mathrm{H}$ mergers of layers forming in the initially uniform gradient-the configuration analogous to that in Fig. 1-and in the two-layer systems (counterparts of the foregoing experiments 1-4).

The significance of merging events lies in the possibility that mergers control the equilibrium structure of staircases (Kelley 1988; Radko 2005). It was hypothesized that relatively small steps are susceptible to merging instabilities, but as their size reaches a critical value, the staircase becomes stable and mergers cease (Radko 2005). The proposed equilibration mechanism is associated with the systematic drift of the interfacial density ratios toward larger values, which has a stabilizing effect on a staircase. The merging simulations examined here cannot offer a definitive confirmation of the equilibration hypothesis because we operate in the strongly mergerfavorable regime. The sizes of the numerical steps are less, by an order of magnitude, than that in the corresponding observations [e.g., $\sim 20 \mathrm{~m}$ in the fingering Caribbean-Sheets and Layers Transect (C-SALT) staircase and $\sim 3 \mathrm{~m}$ in the diffusive staircase in the Beaufort Gyre]. Nevertheless, it is of interest to determine whether the anticipated merger-induced upward drift of the interfacial density ratios is realized in DNS. Therefore, for each experiment we compute the interfacial density ratios prior to and following the merger (Table 1). The operational definition of an interface for these diagnostics is based on the horizontally averaged density gradient, which was required to exceed a threshold value in the interfaces. In all experiments, we find that the postmerger interfacial density ratio exceeds the premerger value, which tends to support the equilibration hypothesis (Radko 2005). Note that the background density ratio remains fixed throughout the simulations, and therefore the drift of interfacial values points toward the significance of the inhomogeneity of the convecting layers for merging dynamics.

\section{c. Validation of theoretical models}

To be more quantitative in the analysis of merging events, the growth rates derived from the simulations $\lambda_{\text {num }}$ are now compared to the theoretical expression $\lambda_{\text {theor }}$ in (2) and to its approximation based on the fourthirds flux law $\lambda_{4 / 3}$ in (5). The numerical growth rates were determined by fitting the exponential pattern (10) to the time records of the merging perturbations $\Delta T_{s w}(t)$ in each experiment.

The estimate of the theoretical growth rate is more involved. It requires knowledge of the "one step" fluxes in (1). The response of fluxes to changes in the temperature and salinity jumps $(\tilde{T}, \tilde{S})$ was evaluated using one-step experiments with different $\tilde{T}$ and $\tilde{S}$, as shown in Fig. 10a. For each of the two-step merging experiments $1-4$ described in sections $3 a$ and $3 b$, we performed four auxiliary one-step simulations, for a total of 16 onestep runs. The resolution and horizontal domain size of the one-step runs was identical to that used for the corresponding two-step experiment (Table 1). The vertical extent of the domain was halved, and therefore the step size was identical to the premerger step height in 
(a)
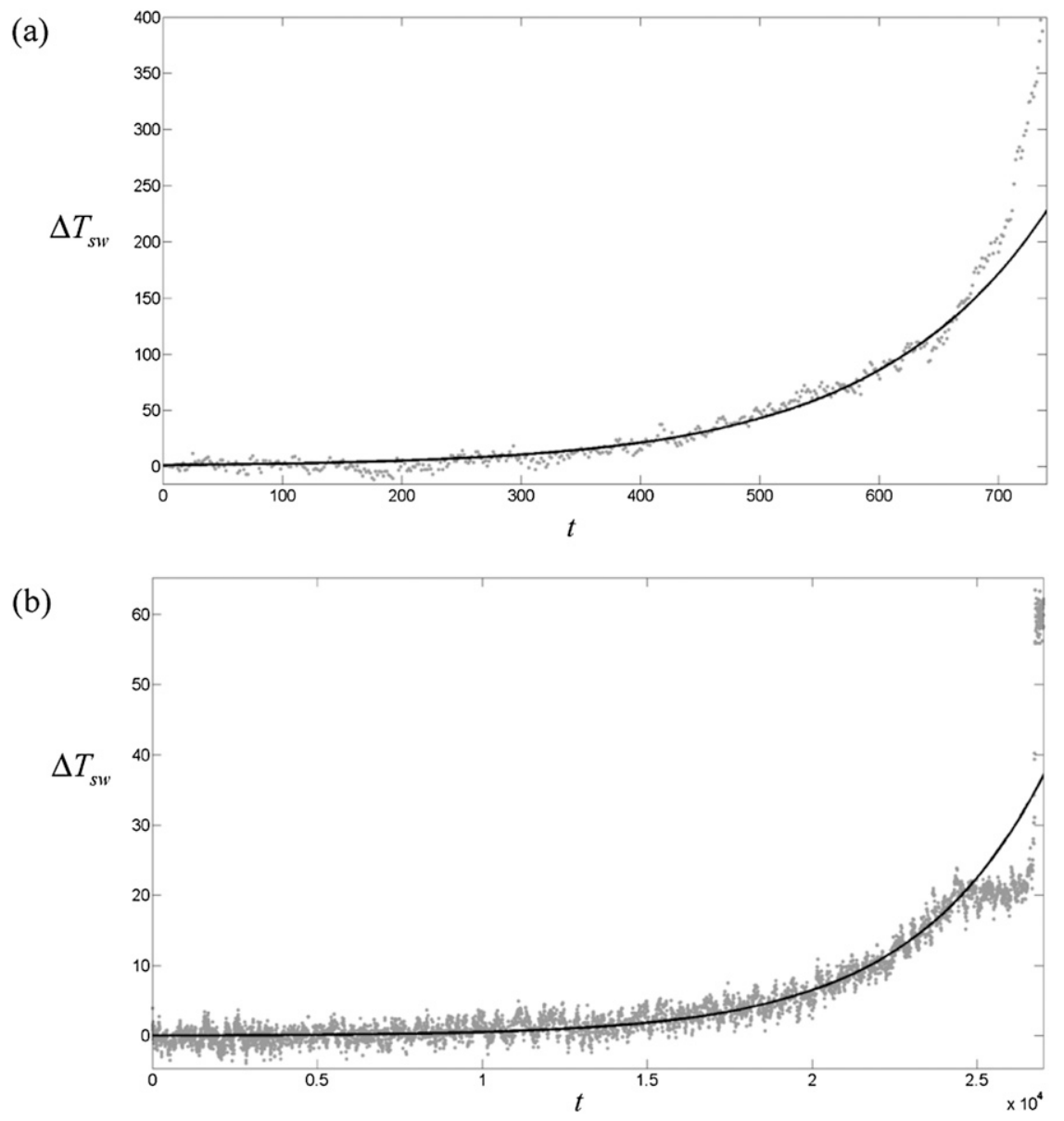

FIG. 8. The strength of merging perturbation $\Delta T_{s w}$ is plotted as a function of $t$ for (a) experiment 1 , representing $2 \mathrm{D}$ fingering and for (b) experiment 2 , the $2 \mathrm{D}$ diffusive case. The solid curves represent the exponential fits to the DNS data evaluated during the linear phase of the instability growth. The total vertical temperature variation across the computational domain is $\Delta T_{\mathrm{tot}}=\bar{T}_{z} L_{z}=400$ for experiment 1 and $\Delta T_{\mathrm{tot}}=60$ for experiment 2.

the two-step experiments. The prescribed overall temperature and salinity variations differed slightly from the initial $T-S$ jumps in the corresponding two-layer experiments, as shown in Fig. 10b. Thus, for a given two-step experiment with the cross-step $T-S$ variation of $\left(\tilde{T}_{0}, \tilde{S}_{0}\right)$, the four auxiliary runs used $(\tilde{T}, \tilde{S})=\left(\tilde{T}_{0}+\Delta T, \tilde{S}_{0}\right)$, $\left(\tilde{T}_{0}-\Delta T, \tilde{S}_{0}\right),\left(\tilde{T}_{0}, \tilde{S}_{0}+\Delta S\right)$, and $\left(\tilde{T}_{0}, \tilde{S}_{0}-\Delta S\right)$, where $\Delta T \ll \tilde{T}_{0}$ and $\Delta S \ll \tilde{S}_{0}$. For instance, the two-step experiment 1 (2D fingering) was based on $\left(\tilde{T}_{0}, \tilde{S}_{0}\right)=(200,154)$, and the perturbations used in the four corresponding one-step runs were $(\Delta T, \Delta S)=(5,6)$. All experiments were initiated by uniform total temperature and salinity fields in the mixed layers separated by a discontinuous interface.

In each one-step run, the equilibrium vertical temperature and salinity fluxes $\left(\tilde{F}^{T}, \tilde{F}^{S}\right)$ were evaluated by computing the time averages of fluxes after adjustment to a quasi-steady regime (Fig. 10c). These equilibrium values were used to evaluate the derivatives of the $T-S$ fluxes as follows:

$$
\left\{\begin{array}{l}
\frac{\partial \tilde{F}^{T}}{\partial \tilde{T}} \approx \frac{\tilde{F}^{T}\left(\tilde{T}_{0}+\Delta T, \tilde{S}_{0}\right)-\tilde{F}^{T}\left(\tilde{T}_{0}-\Delta T, \tilde{S}_{0}\right)}{2 \Delta T}, \\
\frac{\partial \tilde{F}^{T}}{\partial \tilde{S}} \approx \frac{\tilde{F}^{T}\left(\tilde{T}_{0}, \tilde{S}_{0}+\Delta S\right)-\tilde{F}^{T}\left(\tilde{T}_{0}, \tilde{S}_{0}-\Delta S\right)}{2 \Delta S}, \\
\frac{\partial \tilde{F}^{S}}{\partial \tilde{T}} \approx \frac{\tilde{F}^{S}\left(\tilde{T}_{0}+\Delta T, \tilde{S}_{0}\right)-\tilde{F}^{S}\left(\tilde{T}_{0}-\Delta T, \tilde{S}_{0}\right)}{2 \Delta T}, \text { and } \\
\frac{\partial \tilde{F}^{S}}{\partial \tilde{S}} \approx \frac{\tilde{F}^{S}\left(\tilde{T}_{0}, \tilde{S}_{0}+\Delta S\right)-\tilde{F}^{S}\left(\tilde{T}_{0}, \tilde{S}_{0}-\Delta S\right)}{2 \Delta S},
\end{array}\right.
$$

which, in turn, were utilized to compute the growth rates in (2). Similarly, the auxiliary one-step experiments have 
(a)
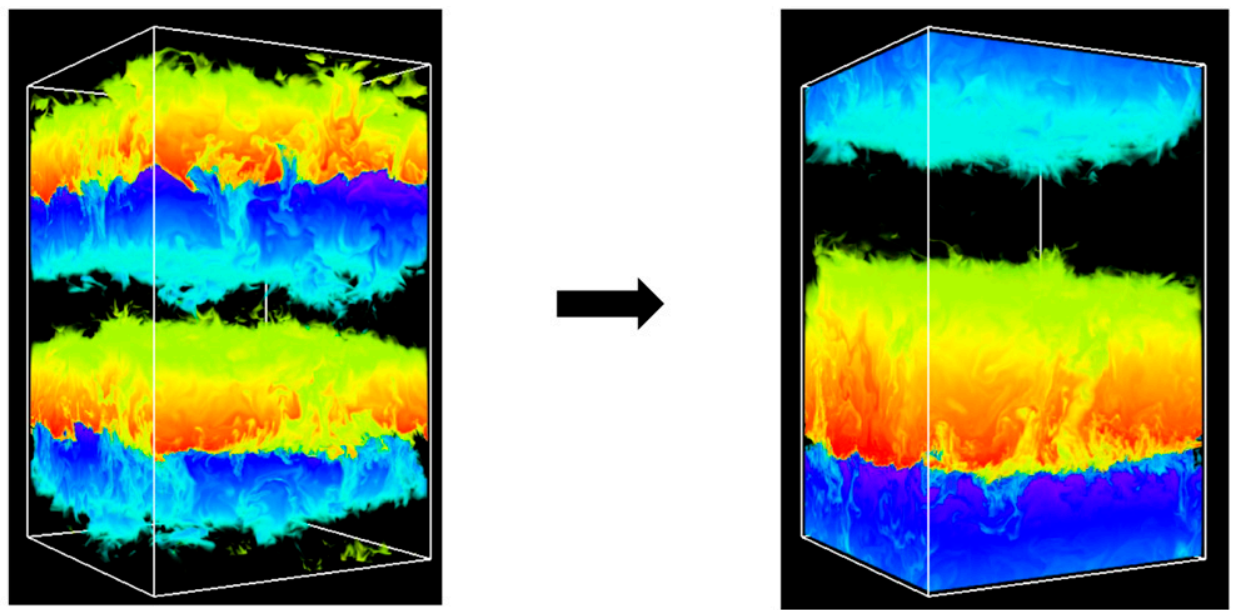

(b)
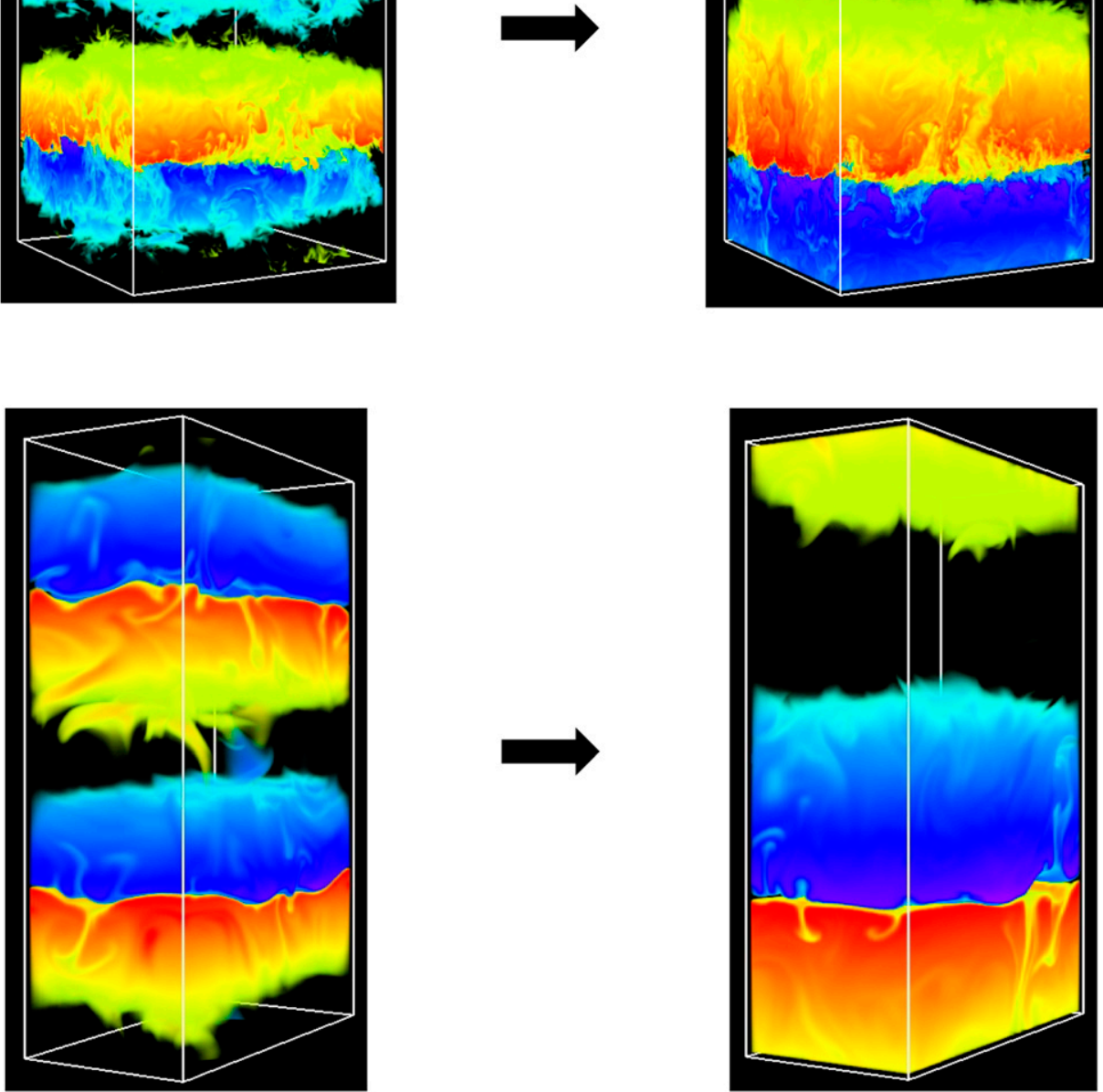

FIG. 9. Three-dimensional merging experiments. (a) The fingering simulation (experiment 3 ) at $t=22$ (premerger) and $t=1893$ (postmerger). (b) The diffusive simulation (experiment 4) for $t=26$ (premerger) and $t=2534$ (postmerger). Visualizations of the temperature perturbation are shown at the (left) early stages of each experiment and in the (right) final, fully merged states.

been used to determine $\partial \gamma / \partial R_{\rho}$ and $\partial C / \partial R_{\rho}$ and thereby to predict the growth rates using the four-thirds law model in (5) and (6).

The results of the growth rate analysis are summarized in Table 2. In all cases, we find that mergers are well described by the general merging theory (2). The estimates based on the simplified expression (3) for the growth rate $\left(\lambda_{\text {theor }_{a}}\right)$ are close to $\lambda_{\text {theor }}$. This suggests that, for most intents and purposes, the full model (2) can be replaced by its approximate but simpler counterpart (3). The prediction (5) based on the four-thirds law is less accurate overall, although it still offers a reasonable estimate of the growth rate with the relative error of $\sim 20 \%$ in most cases. The worst agreement was observed in the three-dimensional diffusive simulation (experiment 4$)$ in which the general theory $\left(\lambda_{\text {theor }}\right)$ overestimated the numerical growth rate by $57 \%$ and the four-thirds law model $\left(\lambda_{4 / 3}\right)$ underestimated it by a factor of 2 .

It should be emphasized that both $\lambda_{\text {theor }}$ and $\lambda_{4 / 3}$ were estimated using numerically determined fluxes, which are dynamically consistent with the two-layer merging experiments. A more interesting and challenging test of merging theory is to determine the growth rates in a 
(a)

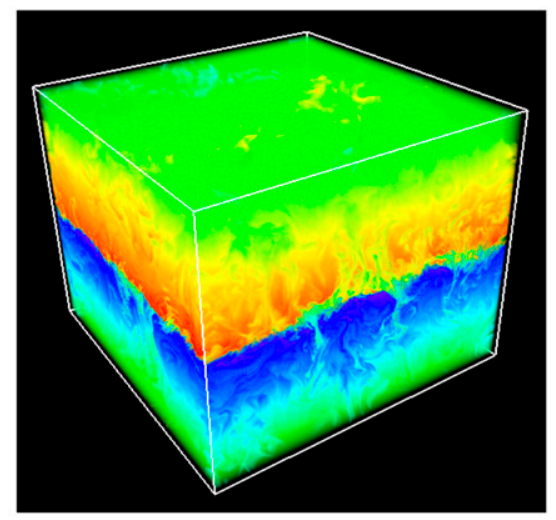

(b)

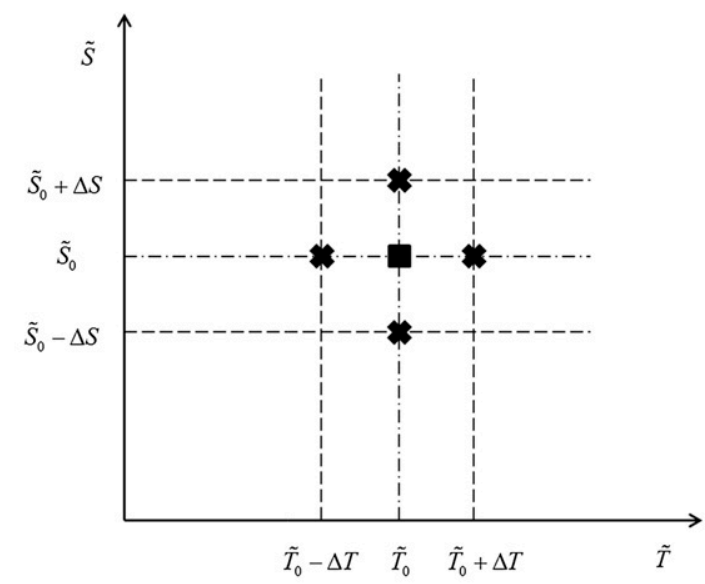

(c)

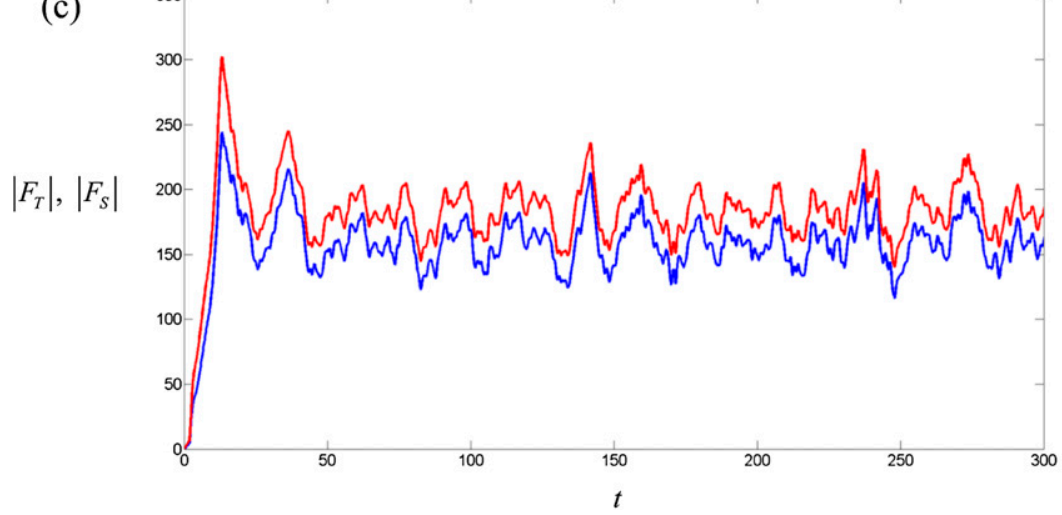

FIG. 10. The setup of one-step experiments used to determine the response of $T-S$ fluxes to changes in the property variations across the interface. (a) Visualization of a typical instantaneous temperature field in a one-step 3D fingering experiment $\left(L_{x}, L_{y}, L_{z}\right)=(335,335,268)$. (b) Selection of the cross-step property variations $(\tilde{T}, \tilde{S})$ in the one-step experiments. For each two-step experiment (indicated by the back square), we perform four auxiliary onestep runs (indicated by the black crosses) with slightly different $\tilde{T}$ and $\tilde{S}$. (c) The time record of the $T-S$ fluxes for the experiment in (a).

completely independent manner, without the information provided by the auxiliary one-step runs. For that we turn to the well-known laboratory-based estimates of flux laws. For fingering interfaces, the laboratory fluxes can be described (Schmitt 1979a; Hamilton et al. 1989) as

$$
\begin{aligned}
g \beta \tilde{F}_{\mathrm{dim}}^{S} & =C_{S}\left(k_{T} g\right)^{1 / 3}\left(\beta \tilde{S}_{\mathrm{dim}}\right)^{4 / 3} \text { and } \\
C_{S} & =0.05+0.3 R_{\rho}^{-3} .
\end{aligned}
$$

The flux ratio pattern $\gamma\left(R_{\rho}\right)$, on the other hand, can be readily estimated using the analytical, fastest-growing finger model (Schmitt 1979b). Numerical simulations (e.g., Radko and Smith 2012) indicate that Schmitt's model offers a qualitatively consistent representation of the flux ratio, overestimating it by only $\sim 10 \%$. Combining this flux ratio model with (12) makes it possible to evaluate the merging growth rate in (5). The resulting estimates $\left(\lambda_{\text {laboratory based }}\right)$ for the salt-finger cases (experiments 1 and 3) are also shown in Table 2. Understandably, these laboratory-based growth rates are less accurate in describing numerical mergers than $\lambda_{\text {theor }}$ or $\lambda_{4 / 3}$. However, they still offer a reasonable (a factor of 2 or better) prediction of merging growth rates.

For the diffusive cases, an independent laboratoryderived flux law is given by Kelley (1990):

$$
\begin{aligned}
\alpha \tilde{F}_{\operatorname{dim}}^{T} & =C_{T}\left(\frac{g k_{T}^{2}}{\nu}\right)^{1 / 3}\left(\alpha \tilde{T}_{\mathrm{dim}}\right)^{4 / 3} \text { and } \\
C_{T} & =0.0032 \exp \left[4.8\left(R_{\rho}^{*}\right)^{-0.72}\right],
\end{aligned}
$$


TABLE 2. The growth rates of simulated mergers along with the corresponding estimates based on the theoretical layer-merging model. Variable $\lambda_{\text {num }}$ represents the growth rate diagnosed from the two-layer DNS; $\lambda_{\text {theor }}$ is based on the most general prediction of the layermerging theory (2); $\lambda_{\text {theor }_{a}}$ is based on its simplified version (3); the estimate $\lambda_{4 / 3}$ assumes that fluxes can be adequately represented by the four-thirds flux law (5) and calibrates those using one-step DNS; and $\lambda_{\text {laboratory based }}$ is also based on the four-thirds flux laws but uses the versions suggested by the laboratory experiments-(12) for fingering and (13) for diffusive convection.

\begin{tabular}{llllll}
\hline \hline & \multicolumn{1}{c}{$\lambda_{\text {theor }}$} & \multicolumn{1}{c}{$\lambda_{\text {theor }_{a}}$} & \multicolumn{1}{c}{$\lambda_{4 / 3}$} & $\lambda_{\text {num }}$ & \multicolumn{1}{c}{$\lambda_{\text {laboratory based }}$} \\
\hline SF2D (Expt 1) & 0.00532 & 0.00569 & 0.00426 & 0.00551 & 0.00352 \\
DC2D (Expt 2) & 0.000235 & 0.000236 & 0.000251 & 0.000240 & 0.00016 \\
SF3D (Expt 3) & 0.00317 & 0.00334 & 0.00313 & 0.00337 & 0.0045 \\
DC3D (Expt 4) & 0.00520 & 0.00555 & 0.00163 & 0.00344 & 0.01625 \\
\hline
\end{tabular}

and the corresponding diffusive flux ratio is

$$
\gamma^{*}=\frac{R_{\rho}^{*}+1.4\left(R_{\rho}^{*}-1\right)^{1.5}}{1+14\left(R_{\rho}^{*}-1\right)^{1.5}} .
$$

Using (5), (13), and (14), we compute $\lambda_{\text {laboratory based }}$ for the diffusive cases (experiments 2 and 4). In the two-dimensional case, $\lambda_{\text {laboratory based }}$ yields an estimate that is sufficiently close to the numerical merger growth rate. However, it is not the case for experiment 4, in which $\lambda_{\text {laboratory based }}$ and $\lambda_{\text {num }}$ differ by a factor of 5 . The reason for the mismatch is attributed to the sensitive dependence of diffusive transport on the diffusivity ratio $\tau$ (e.g., Caro 2009; Carpenter et al. 2012). Fingering convection is less sensitive to $\tau$ (Stern et al. 2001; Radko

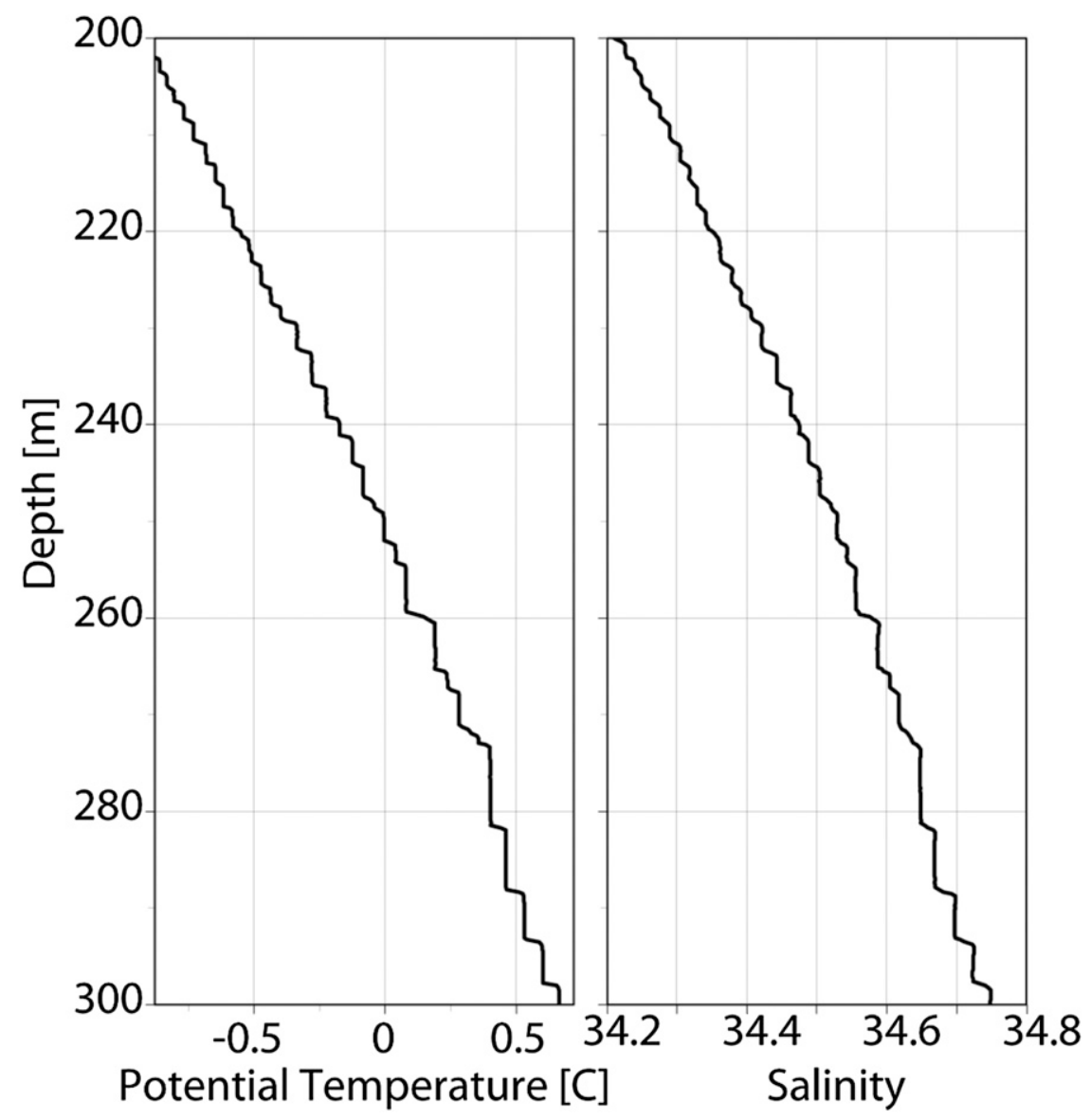

FIG. 11. An example of the $T$ and $S$ profiles recorded by an ITP in the Beaufort Gyre in the diffusive region between 200 and $300 \mathrm{~m}$. Note the well-defined steps consisting of well-mixed layers separated by high-gradient interfaces. 
2008), and therefore the discrepancy between numerical- and laboratory-derived values in the $3 \mathrm{D}$ fingering case (experiment 3 ) is much lower.

\section{Observations}

The foregoing numerical simulations lend credence to theoretical layer-merging models and suggest that the merging framework is ready for application to field measurements. The analysis in this section is based on high-resolution salinity, temperature, and depth measurements made by ITP systems (www.whoi.edu/itp; Toole et al. 2011; Krishfield et al. 2008) operating in the Arctic Ocean's Canada Basin. The ITPs are automated systems designed to profile the upper Arctic Ocean beneath the permanent sea ice cover to a depth of around $750 \mathrm{~m}$. Each system consists of a surface buoy that sits on a sea ice floe and drifts with it. A tether extending beneath the surface buoy supports an underwater profiling unit, cycling vertically up and down the tether to obtain water column measurements of salinity, temperature, and depth. ITPs have proven to be very effective at measuring the properties of a double-diffusive staircase in the Arctic because of their high vertical and horizontal resolution (around $25 \mathrm{~cm}$ and a few kilometers, respectively). Further details of ITP measurements through the staircase and system capabilities with regards to these measurements are given in Timmermans et al. (2008). Here, we do not provide a comprehensive statistical analysis of all existing ITP measurements through the staircase, but rather outline a few examples that appear to support our layer-merging framework.

A double-diffusive staircase is observed in the Canada Basin between around 200- and 300-m depth at the top boundary of a relatively warm and salty layer (of Atlantic origin) that underlies cooler, fresher surface waters. The representative step structure of temperature and salinity in this staircase is shown in Fig. 11. Figures 12 and 13 illustrate what appears to be a layer-merging event measured by one of the ITP systems (ITP 1 ; http://www. whoi.edu/page.do?pid $=28755)$ in the central Canada Basin. Salinity profiles separated by $\Delta t=8.5$ days (Fig. 12a) indicate that, at the same vertical depth range, the number of layers reduces in time because of the merger of two layers. The resultant layer has a middepth around $209.5 \mathrm{~m}$. A time series of salinity at the middepths of each of these two merging layers clearly shows the merger reflected by the systematic drift of two distinct sequences of salinity values toward a single value (Fig. 12b). The evolutionary pattern suggests that the merger occurred via the gradual reduction in strength of an interface that ultimately disappears (i.e., the B merger). Although

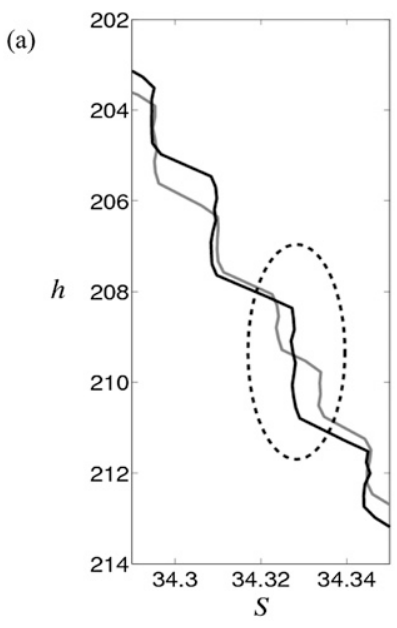

(b)

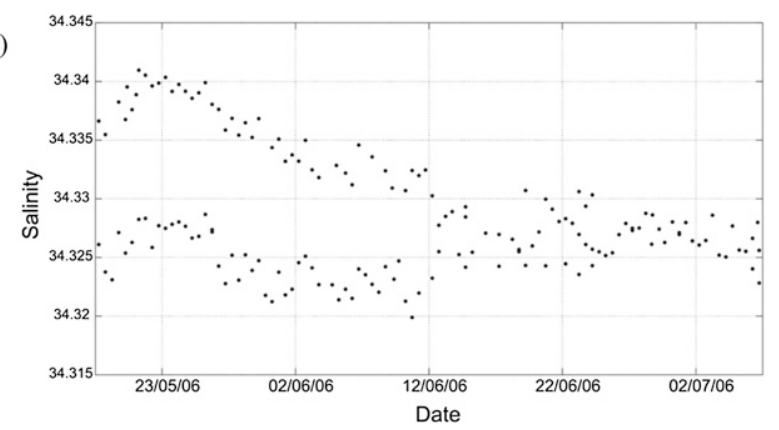

FIG. 12. (a) Profiles of $S$ showing an example of a merging event measured by ITP 1 in the central Canada Basin. Profiles were taken at 0600 UTC 10 Jun (gray) and at 1800 UTC 18 Jun (black) 2006. The dashed oval shows the result of a merger. Note the small vertical offset $(<1 \mathrm{~m})$ between profiles, which is due to vertical excursions of the water column. (b) Time series of $S$ at the centers of the mixed layers above and below the vanishing interface for the event shown in (a). In time, the $S$ difference between adjacent layers reduces to zero, which effectively eliminates the interface, a process referred to as the $\mathrm{B}$ merger.

the profiles are separated laterally by about $30 \mathrm{~km}$, the structure change is not likely the result of this spatial separation; Timmermans et al. (2008) have shown that individual mixed layers in the staircase are coherent over hundreds of kilometers, with well-defined lateral temperature and salinity gradients.

Note that the merging time scale deduced on the basis of ITP measurements is typically on the order of several days. Such short time scales indicate that measurements reflect the final violent stage of merging events, such as that observed in the numerical simulations (Fig. 8b) in which this final stage lasted 4 days. The initial phase of slow linear perturbation growth, which preconditions merging layers for the final stage characterized by rapid disintegration of the weakened interface, is clearly identifiable in simulations (section 3), but may be difficult to detect in the ITP measurements. 


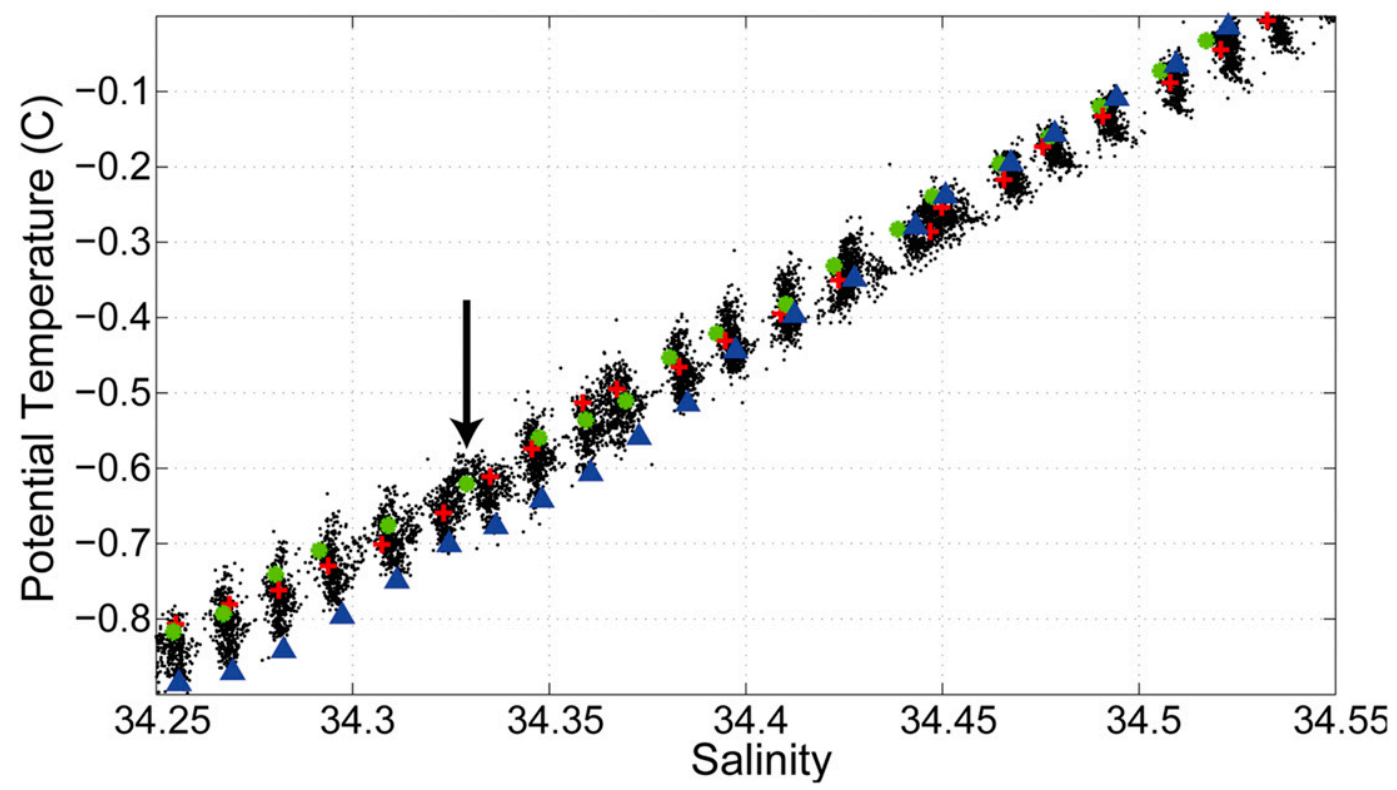

FIG. 13. Potential $T\left({ }^{\circ} \mathrm{C}\right)$ vs $S$ through the staircase measured by ITP 1 ; shown are measurements made between December 2005 and July 2006 in the central Canada Basin, during which time the ITP drifted about $200 \mathrm{~km}$. Each data cluster represents an individual layer (see Timmermans et al. 2008). The example merger (Fig. 12) is reflected by the coalescence of two distinct clusters into a single one (shown by the arrow) that was not present before the merger. Red crosses and green circles represent the layer properties immediately before and after the merger, on 10 and 18 Jun 2006, respectively (gray and black profiles, Fig. 12). Blue triangles show the $T-S$ values from a profile taken on 1 Dec 2005 at the start of the series.

Temperature-salinity diagrams (e.g., Fig. 13) offer yet another dynamic view of merging events. The clusters of points in Fig. 13 correspond to individual mixed layers, coherent across hundreds of kilometers, with welldefined lateral gradients in temperature and salinity (see Timmermans et al. 2008). A merger is evident by the coalescence of two distinct adjacent clusters into a new cluster in temperature-salinity space. The $T-S$ diagrams also illustrate interesting effects that are fundamentally associated with the large-scale lateral variability-effects that are present in the Arctic staircase, but not represented by the relatively small-scale numerical simulations in section 3. Because ITPs drift laterally with an ice floe, the variability of temperature and salinity reflects spatial as well as temporal changes. Observations reveal a trend associated with ITP drift into regions with different $T-S$ properties, which is manifested by the elongated appearance of each cluster in the $T-S$ diagram (Fig. 13). The combination of the merginginduced coalescence of clusters in $T-S$ space and the systematic trend induced by lateral ITP drift show up as characteristic $\mathrm{Y}$-shaped patterns in $T-S$ diagrams. If the merger occurs near the end of the time series, as in Fig. 13a, a V-shaped feature is observed. These $T-S$ patterns have been used for preliminary identification of mergers in ITP data.
The analysis of mergers leads us to yet another potentially important conclusion. The observed mergers, which are recorded in Table 3, are characterized by relatively small step heights and property variations ( $H, \Delta T_{0}$, and $\left.\Delta S_{0}\right)$. This feature is illustrated by comparing $H, \Delta T_{0}$, and $\Delta S_{0}$ for each merger with the corresponding average values $\left(H_{\text {avg }}, \Delta T_{\text {avg }}\right.$, and $\left.\Delta S_{\text {avg }}\right)$, for comparable density ratios $R_{\rho}^{*}$. In all cases, we find that $H<H_{\text {avg }}, \Delta T_{0}<\Delta T_{\text {avg }}$, and $\Delta S_{0}<\Delta S_{\text {avg. This }}$ can be viewed as a sign that mergers effectively regulate step heights and property variations in response to long-term changes in the environment. They eliminate small steps in a staircase and thereby increase the average layer thickness by engaging the familiar "survival of the fittest/ demise of the weakest" dynamics. The importance of these events for regulating step characteristics of fingering staircases was advocated in Part I of our study on the basis of large-scale modeling. Given the strong analogies that exist between the diffusive and fingering cases, it is perhaps not surprising that the diffusive Arctic staircases might be governed by the same principles.

\section{Discussion}

The existence of thermohaline staircases-striking series of regular steps in vertical $T-S$ profiles-is firmly 
TABLE 3. The key characteristics of the mergers identified in the ITP data. The second column lists the number of each ITP (www.whoi. edu/itp). Variables $R_{\rho}^{*}, H, \Delta T_{0}$, and $\Delta S_{0}$ are the density ratios, heights, and temperature and salinity variations across the eroding interfaces prior to the merger. Variables $H_{\text {avg }}, \Delta T_{\text {avg }}$, and $\Delta S_{\text {avg }}$ are the corresponding average properties for similar density ratios $\left(R_{\rho}^{*}-0.25, R_{\rho}^{*}+0.25\right)$, calculated from the same ITP set as the observed mergers. The event shown in Figs. 12 and 13 is denoted as merger number 4 in this table.

\begin{tabular}{crccccccc}
\hline \hline Merger & ITP & $R_{\rho}^{*}$ & $H(\mathrm{~m})$ & $H_{\text {avg }}(\mathrm{m})$ & $\Delta T_{0}\left({ }^{\circ} \mathrm{C}\right)$ & $\Delta T_{\text {avg }}\left({ }^{\circ} \mathrm{C}\right)$ & $\Delta S_{0}(\mathrm{psu})$ & $\Delta S_{\text {avg }}(\mathrm{psu})$ \\
\hline 1 & 4 & 3.42 & 2.24 & 4.00 & 0.0315 & 0.0377 & 0.0085 & 0.0105 \\
2 & 11 & 5.08 & 1.99 & 2.32 & 0.0496 & 0.0497 & 0.0149 & 0.0190 \\
3 & 1 & 5.50 & 1.21 & 2.48 & 0.0330 & 0.0373 & 0.0097 & 0.0137 \\
4 & 1 & 7.15 & 1.47 & 1.82 & 0.0249 & 0.0318 & 0.0109 & 0.0134 \\
5 & 3 & 6.28 & 1.66 & 2.46 & 0.0346 & 0.0374 & 0.0104 & 0.0154 \\
\hline
\end{tabular}

linked to the presence of active double-diffusive processes in the ocean (e.g., Schmitt 1994). However, specific mechanisms for staircase formation, evolution, and ultimate equilibration are uncertain and much debated. Particularly intriguing are the questions related to the selection of the thickness of steps in fully developed staircases. One of the hypotheses (Radko 2005, 2007) views the quasi-permanent staircases as being the result of a series of merging events, which systematically increase the height of steps. The merging model assumes that the coarsening is arrested when layers become sufficiently thick. Therefore, observed staircases are interpreted as marginally equilibrated structures whose average step characteristics represent the boundary between the merger-favorable and merger-unfavorable regions in the parameter space. These effects have been observed in the large-scale simulations presented in Part I of our study. The possibility that mergers control the geometry of staircases, and thus their mixing characteristics, motivates a detailed inquiry into the merging dynamics. The present communication (Part II) attempts to explain the mechanics of such events using a combination of analytical arguments, numerical simulations, and observations.

The recipe for the analysis of staircase evolution is provided by the analytical layer-merging model, which, in turn, is based on the stability analysis of a series of identical steps. Mergers occur when relatively weak interfaces, characterized by low temperature and salinity variations, gradually erode and eventually disappear, a process referred to as the $\mathrm{B}$ merger. The layer-merging model finds support in direct numerical simulations that indicate that layer-merging phenomena are ubiquitous in both fingering and diffusive staircases. The layers formed first in DNS are thin and merge sequentially-see Radko (2003) for fingering and Fig. 1 for the diffusive examples. More quantitative analysis (section 3) indicates that the pattern and time scale of the simulated merging events are consistent with the layer-merging theory. DNS make it possible to evaluate the accuracy of its various versions. For instance, we show that the merging model based on the four-thirds flux laws offers adequate prediction of the merging growth rates. However, it is less accurate than the new model that does not assume such laws a priori and deduces the flux dependencies from one-step simulations.

While it is straightforward to examine the formation of staircases in numerical models, the real world is less accommodating. Most oceanic staircases are in the quasiequilibrium state, and the initial phase of layering-the formation of layers from smooth stratification and systematic increase of their thickness-has not been recorded. However, the ITP observations in the Beaufort Gyre of the Arctic Ocean reveal occasional layermerging events, most likely triggered by changes in the large-scale environmental conditions. The analysis of the continuous temperature and salinity time series recorded by the ITPs suggests that the patterns of observed mergers generally conform to the prediction of the analytical layer-merging model. The mergers act preferentially on relatively thin layers, and therefore in the long run they can effectively regulate the quasiequilibrium structure of the staircase. All mergers identified in this study occurred when the thickness of merging layers $H$ was less than the average thickness of layers with the same density ratio $H_{\mathrm{avg}}\left(R_{\rho}^{*}\right)$. Another suggestive conclusion from the field measurements is that mergers follow the B-merger scenario-the gradual erosion of relatively weak interfaces. Future work will build on these explorative observational results and focus on robust statistical analyses of the entire ITP dataset.

Acknowledgments. The authors thank the editor Karen Heywood and the anonymous reviewers for helpful comments. Support of the National Science Foundation (Grants OCE 1334914, CBET 0933057, and ANT 0944536) is gratefully acknowledged. The ice-tethered profiler data were collected and made available by the Ice-Tethered Profiler Program based at the Woods Hole Oceanographic Institution (www.whoi.edu/itp). The computing 
resources for this project were supplied by the Extreme Science and Engineering Discovery Environment (XSEDE) program, which is supported by the National Science Foundation Grant OCI-1053575.

\section{APPENDIX}

\section{Growth Rate of Merging Events}

The following is an outline of the one-dimensional theory for layer-merging events in a thermohaline staircase that extends the Radko (2007) model to systems with two distinct density components. We examine the stability of the steady state consisting of identical steps of height $H$ (Fig. 2a). We are concerned by balanced perturbations to that state- perturbations that do not affect the background temperature and salinity gradients. Thus, a slight decrease in temperature variation across one of the interfaces (Fig. 2b) is compensated for by an equivalent increase across the other,

$$
\left\{\begin{array}{l}
T_{1,2}-T_{0,1}=T_{0}-\delta \\
T_{2,3}-T_{1,2}=T_{0}+\delta,
\end{array}|\delta| \ll\left|T_{0}\right|,\right.
$$

and the same holds for salinity,

$$
\left\{\begin{array}{l}
S_{1,2}-S_{0,1}=S_{0}-\varepsilon \\
S_{2,3}-S_{1,2}=S_{0}+\varepsilon .
\end{array}|\varepsilon| \ll\left|S_{0}\right|,\right.
$$

where $T_{n, n+1}\left(S_{n, n+1}\right)$ denotes the value of temperature (salinity) at the level exactly between two adjacent interfaces $z_{n, n+1}=0.5\left(z_{n}+z_{n+1}\right)$. The equilibrium jumps, $T_{0}=\bar{T}_{z} H$ and $S_{0}=\bar{S}_{z} H$ in (A1) and (A2), could be positive (for the salt-finger case) or negative (for the diffusive case). The system of layers and interfaces in Fig. 2 is periodic with the $z$ wavelength of $2 H$.

In principle, it is possible to consider, in a similar manner, the merging instabilities resulting in the vertical drift of the interfaces (Radko 2007) by allowing balanced changes in step heights (Fig. 2b). The associated unstable modes (classified as $\mathrm{H}$ instabilities in Radko 2007) are driven by the (weak) variation of the interfacial $T-S$ fluxes with step height. As a result, $\mathrm{H}$ instabilities are characterized by much lower growth rates. The detailed analysis of $\mathrm{H}$ mergers will not be pursued here because measurements and simulations considered in this study suggest a preponderance of B mergers. Therefore, we assume that depths of adjacent layers $(H)$ are equal and constant in time.

The following stability analysis is based on the onedimensional conservation equations for temperature and salinity:

$$
\left\{\begin{array}{l}
\frac{d T}{d t}=\frac{d F^{T}}{d z} \text { and } \\
\frac{d S}{d t}=\frac{d F^{S}}{d z} .
\end{array}\right.
$$

Integrating (A3) over the interval from $z_{n}$ to $z_{n+1}$ between two adjacent interfaces results in

$$
\left\{\begin{array}{l}
\frac{d}{d t} \int_{z_{n}}^{z_{n+1}} T d z=F_{n+1}^{T}-F_{n}^{T} \text { and } \\
\frac{d}{d t} \int_{z_{n}}^{z_{n+1}} S d z=F_{n+1}^{S}-F_{n}^{S}
\end{array}\right.
$$

where $F_{i}=F\left(z_{i}\right)$. The major contribution to the integral of buoyancy in (A4) comes from the interior well-mixed layers, and therefore,

$$
\left\{\begin{array}{l}
\int_{z_{n}}^{z_{n+1}} T d z \approx T_{n, n+1} H \quad \text { and } \\
\int_{z_{n}}^{z_{n+1}} S d z \approx S_{n, n+1} H
\end{array}\right.
$$

where $T_{n, n+1}$ and $S_{n, n+1}$ are the temperature and salinity values at the layer centers $(n=1,2)$. Combining (A1), (A2), (A4), and (A5) leads to the evolutionary equations for $\delta$ and $\varepsilon$, which are then simplified using periodicity conditions:

$$
\left\{\begin{array}{l}
H \frac{d \delta}{d t}=-2\left(F_{2}^{T}-F_{1}^{T}\right) \quad \text { and } \\
H \frac{d \varepsilon}{d t}=-2\left(F_{2}^{S}-F_{1}^{S}\right)
\end{array}\right.
$$

At this point, it becomes necessary to specify how the fluxes in (A6) depend on temperature-salinity jumps across the steps. For that, we replace the instantaneous fluxes in interfaces $F_{n}$ in (A6) with the steady one-step flux laws in (1) based on the parameters of a region extending between the centers of two adjacent layers $\left(z_{n-1, n}\right.$ and $\left.z_{n, n+1}\right)$ :

$$
\left\{\begin{array}{l}
F_{1}^{T} \approx \tilde{F}^{T}\left(T_{1,2}-T_{0,1}, S_{1,2}-S_{0,1}\right) \\
F_{2}^{T} \approx \tilde{F}^{T}\left(T_{2,3}-T_{1,2}, S_{2,3}-S_{1,2}\right) \\
F_{1}^{S} \approx \tilde{F}^{S}\left(T_{1,2}-T_{0,1}, S_{1,2}-S_{0,1}\right) \\
F_{2}^{S} \approx \tilde{F}^{S}\left(T_{1,2}-T_{0,1}, S_{1,2}-S_{0,1}\right) .
\end{array}\right.
$$

For small $(\varepsilon, \delta)$, the difference between fluxes at the adjacent interfaces in (A7) reduces to 


$$
\left\{\begin{array}{l}
F_{2}^{T}-F_{1}^{T}=2 \delta \frac{\partial \tilde{F}^{T}}{\partial \tilde{T}}+2 \varepsilon \frac{\partial \tilde{F}^{T}}{\partial \tilde{S}} \quad \text { and } \\
F_{2}^{S}-F_{1}^{S}=2 \delta \frac{\partial \tilde{F}^{S}}{\partial \tilde{T}}+2 \varepsilon \frac{\partial \tilde{F}^{S}}{\partial \tilde{S}},
\end{array}\right.
$$

and (A6) becomes

$$
\left\{\begin{array}{l}
\frac{d}{d t} \delta=-\frac{4 \delta}{H} \frac{\partial \tilde{F}^{T}}{\partial \tilde{T}}-\frac{4 \varepsilon}{H} \frac{\partial \tilde{F}^{T}}{\partial \tilde{S}} \quad \text { and } \\
\frac{d}{d t} \varepsilon=-\frac{4 \delta}{H} \frac{\partial \tilde{F}^{S}}{\partial \tilde{T}}-\frac{4 \varepsilon}{H} \frac{\partial \tilde{F}^{S}}{\partial \tilde{S}} .
\end{array}\right.
$$

Finally, substitution of the normal modes $(\delta, \varepsilon)=$ $\left(\delta_{0}, \varepsilon_{0}\right) \exp (\lambda t)$ into (A9) yields the eigenvalue equation for the B-merger growth rates:

$$
\begin{aligned}
\lambda^{2} & +\frac{4}{H}\left(\frac{\partial \tilde{F}^{T}}{\partial \tilde{T}}+\frac{\partial \tilde{F}^{S}}{\partial \tilde{S}}\right) \lambda \\
& +\frac{16}{H^{2}}\left(\frac{\partial \tilde{F}^{T}}{\partial \tilde{T}} \frac{\partial \tilde{F}^{S}}{\partial \tilde{S}}-\frac{\partial \tilde{F}^{S}}{\partial \tilde{T}} \frac{\partial \tilde{F}^{T}}{\partial \tilde{S}}\right)=0 .
\end{aligned}
$$

The solution of the quadratic equation (A10) can be simplified by expressing the temperature flux as $\tilde{F}^{T}=$ $(\beta / \alpha) \gamma \tilde{F}^{S}$ and estimating the scales of its coefficients as follows:

$$
\left\{\begin{array}{l}
b=\frac{4}{H}\left(\frac{\partial \tilde{F}^{T}}{\partial \tilde{T}}+\frac{\partial \tilde{F}^{S}}{\partial \tilde{S}}\right) \sim \frac{4}{H} \frac{\tilde{F}^{S}}{\tilde{S}} \quad \text { and } \\
c=\frac{16}{H^{2}}\left(\frac{\partial \tilde{F}^{T}}{\partial \tilde{T}} \frac{\partial \tilde{F}^{S}}{\partial \tilde{S}}-\frac{\partial \tilde{F}^{S}}{\partial \tilde{T}} \frac{\partial \tilde{F}^{T}}{\partial \tilde{S}}\right) \sim \Delta \gamma\left(\frac{4}{H} \frac{\tilde{F}^{S}}{\tilde{S}}\right)^{2}
\end{array}\right.
$$

In deriving (A11), we have assumed that $O(1)$ changes in $\tilde{S}$ or $\tilde{T}$ result in the $O(1)$ response in $\tilde{F}^{S}$ and that $\alpha \tilde{T} \sim \beta \tilde{S}$. The flux ratio, on the other hand, is more constrained, and therefore $\Delta \gamma \ll 1$. Hence, $c \ll b^{2}$ and the discriminant of the quadratic (A11) is positive: $D=b^{2}-4 c>0$. This implies that (A10) has two real roots and that the larger one is given by

$$
\lambda=\frac{-b+\sqrt{b^{2}-4 c}}{2} \approx \frac{c}{b}=\frac{4}{H} \frac{\frac{\partial \tilde{F}^{T}}{\partial \tilde{T}} \frac{\partial \tilde{F}^{S}}{\partial \tilde{S}}-\frac{\partial \tilde{F}^{S}}{\partial \tilde{T}} \frac{\partial \tilde{F}^{T}}{\partial \tilde{S}}}{\frac{\partial \tilde{F}^{T}}{\partial \tilde{T}}+\frac{\partial \tilde{F}^{S}}{\partial \tilde{S}}} .
$$

\section{REFERENCES}

Caro, G. P., 2009: Direct numerical simulations of diffusive staircases in the Arctic. M.S. thesis, Dept. of Oceanography, Naval Postgraduate School, $61 \mathrm{pp}$.

Carpenter, J. R., T. Sommer, and A. Wüest, 2012: Simulations of a double-diffusive interface in the diffusive convection regime. J. Fluid Mech., 711, 411-436, doi:10.1017/ jfm.2012.399.

Flanagan, J. D., A. S. Lefler, and T. Radko, 2013: Heat transport through diffusive interfaces. Geophys. Res. Lett., 40, 24662470, doi:10.1002/grl.50440.

Hamilton, J. M., M. R. Lewis, and B. R. Ruddick, 1989: Vertical fluxes of nitrate associated with salt fingers in the world's oceans. J. Geophys. Res., 94, 2137-2145, doi:10.1029/ JC094iC02p02137.

Huppert, H. E., 1971: On the stability of a series of doublediffusive layers. Deep-Sea Res. Oceanogr. Abstr., 18, 1005-1021, doi:10.1016/0011-7471(71)90005-2.

Kelley, D. E., 1988: Explaining effective diffusivities within diffusive oceanic staircases. Small-Scale Turbulence and Mixing in the Ocean, J. C. J. Nihoul and B. M. Jamart, Eds., Elsevier, 481-502.

— 1990: Fluxes through diffusive staircases: A new formulation. J. Geophys. Res., 95, 3365-3371, doi:10.1029/ JC095iC03p03365.

$\longrightarrow$, H. J. S. Fernando, A. E. Gargett, J. Tanny, and E. Ozsoy, 2003: The diffusive regime of double-diffusive convection. Prog. Oceanogr., 56, 461-481.

Kimura, S., and W. D. Smyth, 2007: Direct numerical simulation of salt sheets and turbulence in a double-diffusive shear layer. Geophys. Res. Lett., 34, L21610, doi:10.1029/ 2007 GL031935.

Krishfield, R., J. Toole, A. Proshutinsky, and M.-L. Timmermans, 2008: Automated ice-tethered profilers for seawater observations under pack ice in all seasons. J. Atmos. Oceanic Technol., 25, 2091-2095, doi:10.1175/2008JTECHO587.1.

Kunze, E., 1987: Limits on growing, finite length salt fingers: A Richardson number constraint. J. Mar. Res., 45, 533-556, doi: $10.1357 / 002224087788326885$.

_ 2003: A review of oceanic salt-fingering theory. Prog. Oceanogr., 56, 399-417.

Linden, P. F., 1974: Salt fingers in a steady shear flow. Geophys. Fluid Dyn., 6, 1-27, doi:10.1080/03091927409365785.

Marmorino, G. O., and D. R. Caldwell, 1976: Heat and salt transport through a diffusive thermohaline interface. Deep-Sea Res. Oceanogr. Abstr., 23, 59-67, doi:10.1016/0011-7471(76)90808-1.

Merryfield, W. J., 2000: Origin of thermohaline staircases. J. Phys. Oceanogr., 30, 1046-1068, doi:10.1175/1520-0485(2000)030<1046: OOTS $>2.0 . \mathrm{CO} ; 2$.

Morell, J. M., J. E. Corredor, and W. J. Merryfield, 2006: Thermohaline staircases in a Caribbean eddy and mechanisms for staircase formation. Deep-Sea Res. II, 53, 128-139, doi:10.1016/ j.dsr2.2005.09.013.

Neal, V. T., S. Neshyba, and W. Denner, 1969: Thermal stratification in the Arctic Ocean. Science, 166, 373-374, doi:10.1126/ science.166.3903.373.

Neshyba, S., V. T. Neal, and W. W. Denner, 1971: Temperature and conductivity measurements under ice island T-3. J. Geophys. Res., 76, 8107-8120, doi:10.1029/JC076i033p08107.

Padman, L., and T. M. Dillon, 1987: Vertical heat fluxes through the Beaufort Sea thermohaline staircase. J. Geophys. Res., 92, 10 799-10 806, doi:10.1029/JC092iC10p10799. 
Park, Y.-G., J. A. Whitehead, and A. Gnanadeskian, 1994: Turbulent mixing in stratified fluids: Layer formation and energetics. J. Fluid Mech., 279, 279-311, doi:10.1017/S0022112094003915.

Radko, T., 2003: A mechanism for layer formation in a doublediffusive fluid. J. Fluid Mech., 497, 365-380, doi:10.1017/ S0022112003006785.

- 2005: What determines the thickness of layers in a thermohaline staircase? J. Fluid Mech., 523, 79-98, doi:10.1017/ S0022112004002290.

— , 2007: Mechanics of merging events for a series of layers in a stratified turbulent fluid. J. Fluid Mech., 577, 251-273, doi:10.1017/S0022112007004703.

— 2008: The double-diffusive modon. J. Fluid Mech., 609, 59-85, doi:10.1017/S0022112008002127.

— , 2013: Double-Diffusive Convection. Cambridge University Press, $344 \mathrm{pp}$

—, and D. P. Smith, 2012: Equilibrium transport in doublediffusive convection. J. Fluid Mech., 692, 5-27, doi:10.1017/ jfm.2011.343.

— A. Bulters, J. Flanagan, and J.-M. Campin, 2014: Doublediffusive recipes. Part I: Large-scale dynamics of thermohaline staircases. J. Phys. Oceanogr., 44, 1269-1284, doi:10.1175/JPO-D-13-0155.1.

Ruddick, B. R., T. J. McDougall, and J. S. Turner, 1989: The formation of layers in a uniformly stirred density gradient. DeepSea Res., 36, 597-609, doi:10.1016/0198-0149(89)90009-5.

Schmitt, R. W., 1979a: Flux measurements on salt fingers at an interface. J. Mar. Res., 37, 419-436.

— 1979b: Flux measurements in an interface. J. Mar. Res., 37, 419-436.

__ 1994: Double diffusion in oceanography. Annu. Rev. Fluid Mech., 26, 255-285, doi:10.1146/annurev.fl.26.010194.001351.
Stellmach, S., A. Traxler, P. Garaud, N. Brummell, and T. Radko, 2011: Dynamics of fingering convection. Part 2: The formation of thermohaline staircases. J. Fluid Mech., 677, 554-571, doi:10.1017/jfm.2011.99.

Stern, M. E., 1969: Collective instability of salt fingers. J. Fluid Mech., 35, 209-218, doi:10.1017/S0022112069001066.

—-, T. Radko, and J. Simeonov, 2001: 3D salt fingers in an unbounded thermocline with application to the central ocean. J. Mar. Res., 59, 355-390, doi:10.1357/ 002224001762842244.

Timmermans, M.-L., J. Toole, R. Krishfield, and P. Winsor, 2008: Ice-tethered profiler observations of the double-diffusive staircase in the Canada Basin thermohaline. J. Geophys. Res., 113, C00A02, doi:10.1029/2008JC004829.

Toole, J. M., R. A. Krishfield, M.-L. Timmermans, and A. Proshutinsky, 2011: The ice-tethered profiler: Argo of the Arctic. Oceanography, 24, 126-135, doi:10.5670/ oceanog.2011.64.

Traxler, A., S. Stellmach, P. Garaud, T. Radko, and N. Brummel, 2011: Dynamics of fingering convection. Part 1: Small-scale fluxes and large-scale instabilities. J. Fluid Mech., 677, 530553.

Turner, J. S., 1965: The coupled turbulent transports of salt and heat across a sharp density interface. Int. J. Heat Mass Transfer, $\mathbf{8}$, 759-767, doi:10.1016/0017-9310(65)90022-0.

_ 1967: Salt fingers across a density interface. Deep-Sea Res. Oceanogr. Abstr., 14, 599-608, doi:10.1016/0011-7471(67)90066-6.

_ 1973: Buoyancy Effects in Fluids. Cambridge University Press, 368 pp.

Zodiatis, G., and G. P. Gasparini, 1996: Thermohaline staircase formations in the Tyrrhenian Sea. Deep-Sea Res., 43, 655-678, doi:10.1016/0967-0637(96)00032-5. 